\title{
A Two-Parameter Defect-Correction Method for Computation of Steady-State Viscoelastic Fluid Flow
}

\author{
Vincent J. Ervin* Jason S. Howell ${ }^{\dagger} \quad$ Hyesuk Lee ${ }^{\ddagger}$
}

\begin{abstract}
The numerical simulation of viscoelastic fluid flow becomes more difficult as a physical parameter, the Weissenberg number, increases. Specifically, at a Weissenberg number larger than a critical value, the iterative nonlinear solver fails to converge. In this paper a two-parameter defect-correction method for viscoelastic fluid flow is presented and analyzed. In the defect step the Weissenberg number is artificially reduced to solve a stable nonlinear problem. The approximation is then improved in the correction step using a linearized correction iteration. Numerical experiments support the theoretical results and demonstrate the effectiveness of the method.
\end{abstract}

Key words. viscoelastic fluid, defect correction, finite element, discontinuous Galerkin, Weissenberg number

AMS Mathematics subject classifications. 65N30

\section{Introduction}

The numerical simulation of viscoelastic fluid flows is a difficult nonlinear problem. This is due mainly to (i) the number of unknowns required for an accurate computation over the problem domain, and (ii) the hyperbolic, nonlinear character of the constitutive equation for the stress. In addition, as the Weissenberg number increases, boundary layers for the stress develop which add to the difficulty of computing accurate numerical approximations. A common difficulty for approximation algorithms is that for high Weissenberg numbers the nonlinear iteration used to compute the approximation fails to converge. Therefore, there has been considerable interest by researchers over the years in developing stable numerical algorithms for high Weissenberg number flows.

One approach for the numerical approximation of difficult nonlinear problems is defect-correction methods [1]. A defect-correction method has two basic steps. The first step uses a stable, nearby problem to form an initial approximation to the solution of the original given problem. The second step (correction step) iteratively improves the defected approximation using residual corrections.

\footnotetext{
*Department of Mathematical Sciences, Clemson University, Clemson, SC, 29634-0975, USA. email: vjervin@clemson.edu. Partially supported by the NSF under grant DMS-0410792.

${ }^{\dagger}$ Department of Mathematical Sciences, Clemson University, Clemson, SC, 29634-0975, USA. email: howell4@clemson.edu. Supported by the NSF under grant DMS-0410792.

${ }^{\ddagger}$ Department of Mathematical Sciences, Clemson University, Clemson, SC, 29634-0975, USA. email: hklee@clemson.edu. Partially supported by the NSF under grant DMS-0410792.
} 
defect-correction methods have been applied convection-diffusion problems $[2,3,4]$ as well as to handle difficulties in computing solutions to the Navier-Stokes equations for high Reynolds number $[5,6]$.

For viscoelastic fluid flows, in [7] Lee presented a method in which the defect step consisted of solving the nonlinear problem for a reduced Weissenberg number on the convective term in the constitutive equation. In the corrector step an iterative, linear residual correction algorithm was used. The error estimate showed that the defect-correction method preserved the optimal order of convergence for the discretization scheme. In [8], a defect-correction method was applied to the linear Oseenviscoelastic model problem. This approach, in the defect step, reduced the Weissenberg number independently on both the convective and stress-deformation interaction terms in the constitutive equation. Again an iterative linear corrector was used.

This manuscript extends the work of [7] and [8]. Specifically, a two-parameter defect-correction algorithm for steady-state viscoelastic fluid flows obeying the nonlinear Johnson-Segalman constitutive model is investigated. To compute solutions for a Weissenberg number larger than the critical value, the original problem is defected by reducing the Weissenberg number to form a nearby, stable problem. These defect parameters can be chosen independently of each other. The initial approximation is then corrected with a linear iteration which, upon convergence, solves the original undefected problem.

The continuation method $[9,10,11]$ can be viewed as an extension of the defect-correction method. In the continuation method, a parameter in the equations is incrementally increased as computations proceed from a readily computable solution to a desired solution. For applications to viscoelastic fluid flow, the Weissenberg number is often used as the continuation parameter $([12,13,14,15,16$, 17,18 ] are some examples). General convergence of continuation methods for viscoelastic fluid flow problems is still an open question.

This paper is organized as follows. In Section 2 the continuous problem and a variational formulation are described in an appropriate setting. A corresponding finite element approximation using the discontinuous Galerkin method is described in Section 3. In Section 4 we present the defect-correction algorithm and show convergence properties of the method. An alternate corrector iteration is also discussed. In Section 5 numerical results are presented to demonstrate the accuracy and effectiveness of the method.

\section{Problem Description}

Let $\Omega$ be a bounded domain in $\mathbb{R}^{d}, d=2$ or 3 , with the Lipschitz continuous boundary $\Gamma$. Consider the viscoelastic (inertialess) fluid flow problem subject to the Johnson-Segalman constitutive equations

$$
\begin{aligned}
\boldsymbol{\sigma}+\lambda(\mathbf{u} \cdot \nabla) \boldsymbol{\sigma}+\lambda g_{a}(\boldsymbol{\sigma}, \nabla \mathbf{u})-2 \alpha \mathbf{D}(\mathbf{u}) & =\mathbf{0} \text { in } \Omega, \\
-\nabla \cdot \boldsymbol{\sigma}-2(1-\alpha) \nabla \cdot \mathbf{D}(\mathbf{u})+\nabla p & =\mathbf{f} \text { in } \Omega, \\
\operatorname{div} \mathbf{u} & =0 \text { in } \Omega, \\
\mathbf{u} & =0 \text { on } \Gamma .
\end{aligned}
$$


In (2.1)-(2.4) $\boldsymbol{\sigma}$ denotes the rank 2 (symmetric) polymeric stress tensor, $\mathbf{u}$ the velocity vector, $p$ the pressure of fluid, and $\lambda$ is the Weissenberg number (defined as the product of the relaxation time of the fluid and a characteristic strain rate). The rank 2 tensor $\nabla \mathbf{u}$ denotes the gradient of $\mathbf{u}$ with components $(\nabla \mathbf{u})_{i, j}=\partial u_{i} / \partial x_{j}$ (consistent with the definition used in $[19,20,21]$ ). Assume that $p$ has zero mean value over $\Omega$. In (2.1) and $(2.2), \mathbf{D}(\mathbf{u}):=\left(\nabla \mathbf{u}+\nabla \mathbf{u}^{T}\right) / 2$ is the rate of the strain tensor, $\alpha \in(0,1)$, and $\mathbf{f}$ the body force. In $(2.1), g_{a}(\boldsymbol{\sigma}, \nabla \mathbf{u})$ is defined by

$$
g_{a}(\boldsymbol{\sigma}, \nabla \mathbf{u}):=\frac{1-a}{2}\left(\boldsymbol{\sigma} \nabla \mathbf{u}+\nabla \mathbf{u}^{T} \boldsymbol{\sigma}\right)-\frac{1+a}{2}\left(\nabla \mathbf{u} \boldsymbol{\sigma}+\boldsymbol{\sigma} \nabla \mathbf{u}^{T}\right)
$$

for $a \in[-1,1]$.

Remark 2.1 For the case $a=1$ the Johnson-Segalman model reduces to the well-known Oldroyd-B model.

\subsection{Continuous Problem}

For $\mathcal{D} \subset \Omega$, denote the standard Sobolev spaces by $W^{m, p}(\mathcal{D})$ with norms $\|\cdot\|_{m, p, \mathcal{D}}$ if $p<\infty,\|\cdot\|_{m, \infty, \mathcal{D}}$ if $p=\infty$. The Sobolev space $W^{m, 2}$ is denoted by $H^{m}$ with the norm $\|\cdot\|_{m}$. The corresponding space of vector-valued or tensor-valued functions is given by $\mathbf{H}^{m}$. If $\mathcal{D}=\Omega, \mathcal{D}$ is omitted, i.e., $(\cdot, \cdot)=(\cdot, \cdot)_{\Omega}$ and $\|\cdot\|=\|\cdot\|_{\Omega}$.

Existence of a solution to the problem (2.1)-(2.4) was shown by Renardy [22] under a small data assumption. (See also [23] and [24].) Specifically, if $\Omega$ has a $C^{\infty}$-smooth boundary and $\mathbf{f}$ is sufficiently regular and small, the problem (2.1)-(2.4) admits a unique bounded solution $(\boldsymbol{\sigma}, \mathbf{u}, p) \in \mathbf{H}^{2}(\Omega) \times$ $\mathbf{H}^{3}(\Omega) \times H^{2}(\Omega)$.

\subsection{Variational Formulation}

Next we define the function spaces for the velocity $\mathbf{u}$, the pressure $p$ and the stress $\boldsymbol{\sigma}$. Let

$$
\begin{aligned}
& \mathbf{X}:=\mathbf{H}_{0}^{1}(\Omega)=\left\{\mathbf{v} \in \mathbf{H}^{1}(\Omega): \mathbf{v}=\mathbf{0} \quad \text { on } \Gamma\right\}, \\
& S:=L_{0}^{2}(\Omega)=\left\{q \in L^{2}(\Omega): \int_{\Omega} q d \Omega=0\right\}, \\
& \boldsymbol{\Sigma}:=\left(L^{2}(\Omega)\right)^{d \times d} \cap\left\{\boldsymbol{\tau}=\left(\tau_{i j}\right): \tau_{i j}=\tau_{j i}, \mathbf{u} \cdot \nabla \boldsymbol{\tau} \in\left(L^{2}(\Omega)\right)^{d \times d}\right\} .
\end{aligned}
$$

We also introduce the divergence free space

$$
\mathbf{V}=\left\{\mathbf{v} \in \mathbf{X}: \int_{\Omega} q \operatorname{div} \mathbf{v} d \Omega=0 \quad \forall q \in S\right\} .
$$

The corresponding variational formulation of (2.1)-(2.4) is obtained by taking the inner product of (2.1)-(2.3) with stress, velocity, and pressure test functions $\boldsymbol{\tau}, \mathbf{v}$, and $q$ respectively. Specifically the variational formulation is: Given $\mathbf{f} \in \mathbf{H}^{-1}(\Omega)$, find $(\boldsymbol{\sigma}, \mathbf{u}, p) \in \mathbf{\Sigma} \times \mathbf{X} \times S$ such that

$$
\begin{aligned}
& (\boldsymbol{\sigma}, \boldsymbol{\tau})+\lambda((\mathbf{u} \cdot \nabla) \boldsymbol{\sigma}, \boldsymbol{\tau})+\lambda\left(g_{a}(\boldsymbol{\sigma}, \nabla \mathbf{u}), \boldsymbol{\tau}\right)-2 \alpha(\mathbf{D}(\mathbf{u}), \boldsymbol{\tau})=0, \quad \forall \boldsymbol{\tau} \in \boldsymbol{\Sigma}, \\
& (\boldsymbol{\sigma}, D(\mathbf{v}))+2(1-\alpha)(\mathbf{D}(\mathbf{u}), D(\mathbf{v}))-(p, \nabla \cdot \mathbf{v})=(\mathbf{f}, \mathbf{v}), \quad \forall \mathbf{v} \in \mathbf{X}, \\
& (q, \nabla \cdot \mathbf{u})=0, \quad \forall q \in S .
\end{aligned}
$$


Using the divergence free space $\mathbf{V}$, the variational formulation (2.5)-(2.7) is equivalent to:

$$
\begin{aligned}
& (\boldsymbol{\sigma}, \boldsymbol{\tau})+\lambda((\mathbf{u} \cdot \nabla) \boldsymbol{\sigma}, \boldsymbol{\tau})+\lambda\left(g_{a}(\boldsymbol{\sigma}, \nabla \mathbf{u}), \boldsymbol{\tau}\right)-2 \alpha(\mathbf{D}(\mathbf{u}), \boldsymbol{\tau})=0, \quad \forall \boldsymbol{\tau} \in \boldsymbol{\Sigma}, \\
& (\boldsymbol{\sigma}, D(\mathbf{v}))+2(1-\alpha)(\mathbf{D}(\mathbf{u}), \mathbf{D}(\mathbf{v}))=(\mathbf{f}, \mathbf{v}), \quad \forall \mathbf{v} \in \mathbf{V} .
\end{aligned}
$$

We introduce the bilinear form $A$ defined on $\boldsymbol{\Sigma} \times \mathbf{X}$ by

$$
A((\boldsymbol{\sigma}, \mathbf{u}),(\boldsymbol{\tau}, \mathbf{v})):=(\boldsymbol{\sigma}, \boldsymbol{\tau})-2 \alpha(\mathbf{D}(\mathbf{u}), \boldsymbol{\tau})+2 \alpha(\boldsymbol{\sigma}, \mathbf{D}(\mathbf{v}))+4 \alpha(1-\alpha)(\mathbf{D}(\mathbf{u}), \mathbf{D}(\mathbf{v})) .
$$

The continuity and coercivity of $A$ is shown in the following lemma.

Lemma 2.1 The bilinear form A defined in (2.8) is continuous and coercive, i.e., a) (continuity) there exists a positive constant $C$ such that

$$
A((\boldsymbol{\sigma}, \mathbf{u}),(\boldsymbol{\tau}, \mathbf{v})) \leq C\|(\boldsymbol{\sigma}, \mathbf{u})\|_{\boldsymbol{\Sigma} \times \mathbf{X}}\|(\boldsymbol{\tau}, \mathbf{v})\|_{\boldsymbol{\Sigma} \times \mathbf{X}}, \quad \forall(\boldsymbol{\tau}, \mathbf{v}) \in \boldsymbol{\Sigma} \times \mathbf{X},
$$

b) (coercivity) there exists a positive constant $C$ such that

$$
A((\boldsymbol{\sigma}, \mathbf{u}),(\boldsymbol{\sigma}, \mathbf{u})) \geq C\|(\boldsymbol{\sigma}, \mathbf{u})\|_{\mathbf{\Sigma} \times \mathbf{X}}^{2}, \quad \forall(\boldsymbol{\sigma}, \mathbf{u}) \in \mathbf{\Sigma} \times \mathbf{X}
$$

Proof: The reader is referred to Lemma 1.1 of [7].

\section{Finite Element Approximation}

Let $T_{h}$ denote a triangulation of $\Omega$ such that $\bar{\Omega}=\left\{\cup K: K \in T_{h}\right\}$. Assume that there exist positive constants $c_{1}, c_{2}$ such that

$$
c_{1} h \leq h_{K} \leq c_{2} \rho_{K}
$$

where $h_{K}$ is the diameter of $K, \rho_{K}$ is the diameter of the greatest ball included in $K$, and $h=$ $\max _{K \in T_{h}} h_{K}$.

Let $P_{k}(K)$ denote the space of polynomials of degree less than or equal to $k$ on $K \in T_{h}$. As was done in [7], define the following finite element spaces, (Taylor-Hood) for the approximation of $(\mathbf{u}, p)$ :

$$
\begin{aligned}
\mathbf{X}^{h} & :=\left\{\mathbf{v} \in \mathbf{X} \cap\left(C^{0}(\bar{\Omega})\right)^{d}:\left.\mathbf{v}\right|_{K} \in P_{2}(K)^{d}, \forall K \in T_{h}\right\}, \\
S^{h} & :=\left\{q \in S \cap C^{0}(\bar{\Omega}):\left.q\right|_{K} \in P_{1}(K), \forall K \in T_{h}\right\} \\
\mathbf{V}^{h} & :=\left\{\mathbf{v} \in \mathbf{X}^{h}:(q, \nabla \cdot \mathbf{v})=0, \forall q \in S^{h}\right\} .
\end{aligned}
$$

For the approximation of the constitutive equation (2.1) the discontinuous Galerkin method is used, where the stress $\boldsymbol{\sigma}$ is approximated in the discontinuous finite element space of piecewise linears:

$$
\boldsymbol{\Sigma}^{h}:=\left\{\boldsymbol{\tau} \in \boldsymbol{\Sigma}:\left.\boldsymbol{\tau}\right|_{K} \in P_{1}(K)^{d \times d}, \forall K \in T_{h}\right\} .
$$


The finite element spaces defined above satisfy the standard approximation properties (see [20] or [25]), i.e., there exists a constant $C$ such that

$$
\begin{gathered}
\inf _{\mathbf{v}^{h} \in \mathbf{X}^{h}}\left\|\mathbf{v}-\mathbf{v}^{h}\right\|_{1} \leq C h^{2}\|\mathbf{v}\|_{3}, \quad \forall \mathbf{v} \in \mathbf{H}^{3}(\Omega), \\
\inf _{q^{h} \in S^{h}}\left\|q-q^{h}\right\|_{0} \leq C h^{2}\|q\|_{2}, \quad \forall q \in H^{2}(\Omega),
\end{gathered}
$$

and

$$
\inf _{\boldsymbol{\tau}^{h} \in \Sigma^{h}}\left\|\boldsymbol{\tau}-\boldsymbol{\tau}^{h}\right\|_{0} \leq C h^{2}\|\boldsymbol{\tau}\|_{2}, \quad \forall \boldsymbol{\tau} \in \mathbf{H}^{2}(\Omega) .
$$

It is also well known that the Taylor-Hood pair $\left(\mathbf{X}^{h}, S^{h}\right)$ satisfies the inf-sup (or $L B B$ ) condition $([20])$,

$$
\inf _{0 \neq q^{h} \in S^{h}} \sup _{\mathbf{0} \neq \mathbf{v}^{h} \in \mathbf{X}^{h}} \frac{\left(q^{h}, \nabla \cdot \mathbf{v}^{h}\right)}{\left\|\mathbf{v}^{h}\right\|_{1}\left\|q^{h}\right\|_{0}} \geq C,
$$

where $C$ is a positive constant independent of $h$.

Below some notation used in [19] is introduced to describe and analyze an approximate solution obtained using the discontinuous Galerkin method. Let

$$
\begin{gathered}
\Gamma^{h}=\left\{\cup \partial K, K \in T_{h}\right\} \backslash \Gamma, \\
\partial K^{-}(\mathbf{u}):=\{\mathbf{x} \in \partial K, \mathbf{u} \cdot \mathbf{n}<0\},
\end{gathered}
$$

where $\partial K$ is the boundary of $K$ and $\mathbf{n}$ is outward unit normal, and

$$
\boldsymbol{\tau}^{ \pm}(\mathbf{u}):=\lim _{\epsilon \rightarrow 0^{ \pm}} \boldsymbol{\tau}(\mathbf{x}+\epsilon \mathbf{u}(\mathbf{x}))
$$

Also, let

$$
\begin{gathered}
(\boldsymbol{\sigma}, \boldsymbol{\tau})_{h}:=\sum_{K \in T_{h}}(\boldsymbol{\sigma}, \boldsymbol{\tau})_{K} \\
\left\langle\boldsymbol{\sigma}^{ \pm}, \boldsymbol{\tau}^{ \pm}\right\rangle_{h, \mathbf{u}}:=\sum_{K \in T_{h}} \int_{\partial K^{-}(\mathbf{u})}\left(\boldsymbol{\sigma}^{ \pm}(\mathbf{u}): \boldsymbol{\tau}^{ \pm}(\mathbf{u})\right)|\mathbf{n} \cdot \mathbf{u}| d s \\
\left\langle\left\langle\boldsymbol{\sigma}^{ \pm}\right\rangle\right\rangle_{h, \mathbf{u}}:=\left\langle\boldsymbol{\sigma}^{ \pm}, \boldsymbol{\sigma}^{ \pm}\right\rangle_{h, \mathbf{u}}^{1 / 2} \\
\|\boldsymbol{\tau}\|_{0, \Gamma^{h}}:=\left(\sum_{K \in T_{h}}\|\boldsymbol{\tau}\|_{0, \partial K}^{2}\right)^{1 / 2}
\end{gathered}
$$

for $\boldsymbol{\sigma}, \boldsymbol{\tau} \in \prod_{K \in T_{h}}\left(L^{2}(K)\right)^{d \times d}$, and

$$
\|\boldsymbol{\xi}\|_{m, h}:=\left(\sum_{K \in T_{h}}\|\boldsymbol{\xi}\|_{m, K}^{2}\right)^{1 / 2}
$$

for $\boldsymbol{\xi} \in \prod_{K \in T_{h}}\left(W^{m, 2}(K)\right)^{d \times d}$. 
Introduce the operator $B^{h}$ on $\mathbf{X}^{h} \times \boldsymbol{\Sigma}^{h} \times \boldsymbol{\Sigma}^{h}$ defined by

$$
B^{h}\left(\mathbf{u}^{h}, \boldsymbol{\sigma}^{h}, \boldsymbol{\tau}^{h}\right):=\left(\left(\mathbf{u}^{h} \cdot \nabla\right) \boldsymbol{\sigma}^{h}, \boldsymbol{\tau}^{h}\right)_{h}+\frac{1}{2}\left(\nabla \cdot \mathbf{u}^{h} \boldsymbol{\sigma}^{h}, \boldsymbol{\tau}^{h}\right)+\left\langle\boldsymbol{\sigma}^{h^{+}}-\boldsymbol{\sigma}^{h^{-}}, \boldsymbol{\tau}^{h^{+}}\right\rangle_{h, \mathbf{u}^{h}} .
$$

Note that the second term vanishes when $\nabla \cdot \mathbf{u}^{h}=0$ (this is not necessarily the case for any $\mathbf{u}^{h} \in \mathbf{X}^{h}$ ). This extra term is used to obtain coercivity of $B^{h}\left(\mathbf{u}^{h}, \cdot, \cdot\right)$. Using integration by parts, $B^{h}$ may be written as

$$
B^{h}\left(\mathbf{u}^{h}, \boldsymbol{\sigma}^{h}, \boldsymbol{\tau}^{h}\right)=-\left(\left(\mathbf{u}^{h} \cdot \nabla\right) \boldsymbol{\tau}^{h}, \boldsymbol{\sigma}^{h}\right)_{h}-\frac{1}{2}\left(\nabla \cdot \mathbf{u}^{h} \boldsymbol{\tau}^{h}, \boldsymbol{\sigma}^{h}\right)+\left\langle\boldsymbol{\sigma}^{h^{-}}, \boldsymbol{\tau}^{h^{-}}-\boldsymbol{\tau}^{h^{+}}\right\rangle_{h, \mathbf{u}^{h}} .
$$

Combining (3.5) and (3.6), we obtain

$$
B^{h}\left(\mathbf{u}^{h}, \boldsymbol{\sigma}^{h}, \boldsymbol{\sigma}^{h}\right)=\frac{1}{2}\left\langle\boldsymbol{\sigma}^{h^{+}}-\boldsymbol{\sigma}^{h^{-}}, \boldsymbol{\sigma}^{h^{+}}-\boldsymbol{\sigma}^{h^{-}}\right\rangle_{h, \mathbf{u}^{h}}=\frac{1}{2}\left\langle\left\langle\boldsymbol{\sigma}^{h^{+}}-\boldsymbol{\sigma}^{h^{-}}\right\rangle\right\rangle_{h, \mathbf{u}^{h}}^{2} \geq 0 .
$$

The discontinuous Galerkin finite element approximation of (2.5)-(2.7) is then as follows. Given $\mathbf{f} \in \mathbf{H}^{-1}(\Omega)$, find $\left(\boldsymbol{\sigma}^{h}, \mathbf{u}^{h}, p^{h}\right) \in \boldsymbol{\Sigma}^{h} \times \mathbf{X}^{h} \times S^{h}$ such that

$$
\begin{aligned}
& \left(\boldsymbol{\sigma}^{h}, \boldsymbol{\tau}^{h}\right)+\lambda B^{h}\left(\mathbf{u}^{h}, \boldsymbol{\sigma}^{h}, \boldsymbol{\tau}^{h}\right)+\lambda\left(g_{a}\left(\boldsymbol{\sigma}^{h}, \nabla \mathbf{u}^{h}\right), \boldsymbol{\tau}^{h}\right)-2 \alpha\left(\mathbf{D}\left(\mathbf{u}^{h}\right), \boldsymbol{\tau}^{h}\right)=0, \quad \forall \boldsymbol{\tau}^{h} \in \boldsymbol{\Sigma}^{h}, \\
& \left(\boldsymbol{\sigma}^{h}, \mathbf{D}\left(\mathbf{v}^{h}\right)\right)+2(1-\alpha)\left(\mathbf{D}\left(\mathbf{u}^{h}\right), \mathbf{D}\left(\mathbf{v}^{h}\right)\right)-\left(p^{h}, \nabla \cdot \mathbf{v}^{h}\right)=\left(\mathbf{f}, \mathbf{v}^{h}\right), \quad \forall \mathbf{v}^{h} \in \mathbf{X}^{h}, \\
& \left(q^{h}, \nabla \cdot \mathbf{u}^{h}\right)=0, \quad \forall q^{h} \in S^{h} .
\end{aligned}
$$

Existence of a solution to the discrete problem (3.8)-(3.10) has been shown by Baranger and Sandri [19] under the assumption that the continuous problem (2.1)-(2.4) yields a bounded solution $(\mathbf{u}, \boldsymbol{\sigma}, p) \in \mathbf{H}^{3}(\Omega) \times \mathbf{H}^{2}(\Omega) \times H^{2}(\Omega)$. The error estimates

$$
\left\|\boldsymbol{\sigma}-\boldsymbol{\sigma}^{h}\right\|_{0}+\left\|\nabla\left(\mathbf{u}-\mathbf{u}^{h}\right)\right\|_{0} \leq C h^{3 / 2}, \quad\left\|p-p^{h}\right\|_{0} \leq C h^{3 / 2}
$$

for constant $C>0$, are also proven in [19].

Notice that, in view of (3.4), (3.8)-(3.10) is equivalent to: Given $\mathbf{f} \in \mathbf{H}^{-1}(\Omega)$, find $\left(\boldsymbol{\sigma}^{h}, \mathbf{u}^{h}\right) \in \boldsymbol{\Sigma}^{h} \times \mathbf{V}^{h}$ such that

$$
\begin{aligned}
\left(\boldsymbol{\sigma}^{h}, \boldsymbol{\tau}^{h}\right)+\lambda B^{h}\left(\mathbf{u}^{h}, \boldsymbol{\sigma}^{h}, \boldsymbol{\tau}^{h}\right)+\lambda\left(g_{a}\left(\boldsymbol{\sigma}^{h}, \nabla \mathbf{u}^{h}\right), \boldsymbol{\tau}^{h}\right)-2 \alpha\left(\mathbf{D}\left(\mathbf{u}^{h}\right), \boldsymbol{\tau}^{h}\right)=0, & \forall \boldsymbol{\tau}^{h} \in \boldsymbol{\Sigma}^{h}, \\
\left(\boldsymbol{\sigma}^{h}, \mathbf{D}\left(\mathbf{v}^{h}\right)\right)+2(1-\alpha)\left(\mathbf{D}\left(\mathbf{u}^{h}\right), \mathbf{D}\left(\mathbf{v}^{h}\right)\right)=\left(\mathbf{f}, \mathbf{v}^{h}\right), & \forall \mathbf{v}^{h} \in \mathbf{V}^{h} .
\end{aligned}
$$

Using the bilinear form $A$ defined by (2.8), (3.11)-(3.12) can equivalently be written as

$$
\begin{aligned}
A\left(\left(\boldsymbol{\sigma}^{h}, \mathbf{u}^{h}\right),\left(\boldsymbol{\tau}^{h}, \mathbf{v}^{h}\right)\right)+\lambda B^{h}\left(\mathbf{u}^{h}, \boldsymbol{\sigma}^{h}, \boldsymbol{\tau}^{h}\right)+\lambda\left(g_{a}\left(\boldsymbol{\sigma}^{h}, \nabla \mathbf{u}^{h}\right), \boldsymbol{\tau}^{h}\right) & \\
& =2 \alpha\left(\mathbf{f}, \mathbf{v}^{h}\right) \quad \forall\left(\boldsymbol{\tau}^{h}, \mathbf{v}^{h}\right) \in \mathbf{\Sigma}^{h} \times \mathbf{V}^{h} .
\end{aligned}
$$

In the following analysis several inverse estimates are used. For convenience, these results are summarized here. (See [20], [25]). Assume $d=2$. For $\mathbf{u}^{h} \in \mathbf{V}^{h}$ and $\boldsymbol{\sigma}^{h} \in \boldsymbol{\Sigma}^{h}$, we have

$$
\begin{aligned}
\left\|\mathbf{u}^{h}\right\|_{\infty} & \leq C h^{-1 / 2}\left\|\mathbf{u}^{h}\right\|_{0,4}, \\
\left\|\boldsymbol{\sigma}^{h}\right\|_{\infty} & \leq C h^{-1}\left\|\boldsymbol{\sigma}^{h}\right\|_{0}, \\
\left\|\nabla \boldsymbol{\sigma}^{h}\right\|_{0,4, h} & \leq C h^{-3 / 2}\left\|\boldsymbol{\sigma}^{h}\right\|_{0}, \\
\left\|\nabla \boldsymbol{\sigma}^{h}\right\|_{0,2, h} & \leq C h^{-1}\left\|\boldsymbol{\sigma}^{h}\right\|_{0} .
\end{aligned}
$$


The local inverse inequality ([26]),

$$
\|\boldsymbol{\sigma}\|_{0, \partial K}^{2} \leq C \frac{p_{K}^{2}}{h_{K}}\|\boldsymbol{\sigma}\|_{0, K}^{2}
$$

is used to bound the jump term of $B^{h}$, where $p_{K}$ denotes the polynomial degree on mesh element $K$ and $h_{K}$ the local mesh parameter.

\section{Defect Correction Method}

In this section the defect correction method used in computing the solution to (3.8)-(3.10) is described. The idea behind the method is to avoid the approximation difficulties associated with a high Weissenberg number, $\lambda$-difficult, by computing an initial approximation using a $\lambda$-easy value, and then iteratively improving the approximation until the desired solution at $\lambda=\lambda$-difficult is obtained.

\subsection{Algorithm}

To avoid the computational difficulties associated with the high Weissenberg number problem, replace $\lambda$ in the second and third terms of (3.8) with the parameters $\bar{\lambda}$ and $\widetilde{\lambda}$, respectively. These defect parameters are chosen so that $0 \leq \bar{\lambda}, \widetilde{\lambda} \leq \lambda$. Let $\left(\boldsymbol{\sigma}_{0}^{h}, \mathbf{u}_{0}^{h}, p_{0}^{h}\right) \in \boldsymbol{\Sigma}^{h} \times \mathbf{X}^{h} \times S^{h}$ satisfy

$$
\begin{aligned}
& \left(\boldsymbol{\sigma}_{0}^{h}, \boldsymbol{\tau}^{h}\right)+\bar{\lambda} B^{h}\left(\mathbf{u}_{0}^{h}, \boldsymbol{\sigma}_{0}^{h}, \boldsymbol{\tau}^{h}\right)+\widetilde{\lambda}\left(g_{a}\left(\boldsymbol{\sigma}_{0}^{h}, \nabla \mathbf{u}_{0}^{h}\right), \boldsymbol{\tau}^{h}\right)-2 \alpha\left(\mathbf{D}\left(\mathbf{u}_{0}^{h}\right), \boldsymbol{\tau}^{h}\right)=0, \quad \forall \boldsymbol{\tau}^{h} \in \boldsymbol{\Sigma}^{h}, \\
& \left(\boldsymbol{\sigma}_{0}^{h}, \mathbf{D}\left(\mathbf{v}^{h}\right)\right)+2(1-\alpha)\left(\mathbf{D}\left(\mathbf{u}_{0}^{h}\right), \mathbf{D}\left(\mathbf{v}^{h}\right)\right)-\left(p_{0}^{h}, \nabla \cdot \mathbf{v}^{h}\right)=\left(\mathbf{f}, \mathbf{v}^{h}\right), \quad \forall \mathbf{v}^{h} \in \mathbf{X}^{h}, \\
& \left(q^{h}, \nabla \cdot \mathbf{u}_{0}^{h}\right)=0, \quad \forall q^{h} \in S^{h} .
\end{aligned}
$$

Then the residuals $\left(R_{1}, R_{2}, R_{3}\right) \in \boldsymbol{\Sigma}^{h} \times \mathbf{X}^{h} \times S^{h}$ for equations (3.8)-(3.10) are defined by

$$
\begin{aligned}
& \left(R_{1}\left(\mathbf{u}_{0}^{h}, \boldsymbol{\sigma}_{0}^{h}\right), \boldsymbol{\tau}^{h}\right) \\
& \quad:=-\left(\boldsymbol{\sigma}_{0}^{h}, \boldsymbol{\tau}^{h}\right)-\lambda B^{h}\left(\mathbf{u}_{0}^{h}, \boldsymbol{\sigma}_{0}^{h}, \boldsymbol{\tau}^{h}\right)-\lambda\left(g_{a}\left(\boldsymbol{\sigma}_{0}^{h}, \nabla \mathbf{u}_{0}^{h}\right), \boldsymbol{\tau}^{h}\right)+2 \alpha\left(\mathbf{D}\left(\mathbf{u}_{0}^{h}\right), \boldsymbol{\tau}^{h}\right), \quad \forall \boldsymbol{\tau}^{h} \in \boldsymbol{\Sigma}^{h}, \\
& \left(R_{2}\left(\mathbf{u}_{0}^{h}, \boldsymbol{\sigma}_{0}^{h}, p_{0}^{h}\right), \mathbf{v}^{h}\right) \\
& \quad:=\left(\mathbf{f}, \mathbf{v}^{h}\right)-\left(\boldsymbol{\sigma}_{0}^{h}, \mathbf{D}\left(\mathbf{v}^{h}\right)\right)-2(1-\alpha)\left(\mathbf{D}\left(\mathbf{u}_{0}^{h}\right), \mathbf{D}\left(\mathbf{v}_{0}^{h}\right)\right)+\left(p_{0}^{h}, \nabla \cdot \mathbf{v}^{h}\right), \quad \forall \mathbf{v}^{h} \in \mathbf{X}^{h}, \\
& \left(R_{3}\left(\mathbf{u}_{0}^{h}\right), q^{h}\right):=-\left(q^{h}, \nabla \cdot \mathbf{u}_{0}^{h}\right), \quad \forall q^{h} \in S^{h} .
\end{aligned}
$$

We define the correction $\left(\boldsymbol{\xi}_{0}^{h}, \boldsymbol{\epsilon}_{0}^{h}, \rho_{0}^{h}\right) \in \boldsymbol{\Sigma}^{h} \times \mathbf{X}^{h} \times S^{h}$ to the approximation $\left(\boldsymbol{\sigma}_{0}^{h}, \mathbf{u}_{0}^{h}, p_{0}^{h}\right)$ via

$$
\begin{aligned}
& \left.\left(\boldsymbol{\xi}_{0}^{h}, \boldsymbol{\tau}^{h}\right)+\bar{\lambda} B^{h}\left(\mathbf{u}_{0}^{h}, \boldsymbol{\xi}_{0}^{h}, \boldsymbol{\tau}^{h}\right)+\widetilde{\lambda}\left(g_{a}\left(\boldsymbol{\xi}_{0}^{h}, \nabla \mathbf{u}_{0}^{h}\right), \boldsymbol{\tau}^{h}\right)-2 \alpha\left(D\left(\boldsymbol{\epsilon}_{0}^{h}\right), \boldsymbol{\tau}^{h}\right)\right) \\
& =\left(R_{1}\left(\mathbf{u}_{0}^{h}, \boldsymbol{\sigma}_{0}^{h}\right), \boldsymbol{\tau}^{h}\right), \quad \forall \boldsymbol{\tau}^{h} \in \boldsymbol{\Sigma}^{h}, \\
& \left(\boldsymbol{\xi}_{0}^{h}, \mathbf{D}\left(\mathbf{v}^{h}\right)\right)+2(1-\alpha)\left(D\left(\boldsymbol{\epsilon}_{0}^{h}\right), \mathbf{D}\left(\mathbf{v}^{h}\right)\right)-\left(\rho_{0}^{h}, \nabla \cdot \mathbf{v}^{h}\right)=\left(R_{2}\left(\mathbf{u}_{0}^{h}, \boldsymbol{\sigma}_{0}^{h}, p_{0}^{h}\right), \mathbf{v}^{h}\right), \quad \forall \mathbf{v}^{h} \in \mathbf{X}^{h}, \\
& \left(q^{h}, \nabla \cdot \epsilon_{0}^{h}\right)=\left(R_{3}\left(\mathbf{u}_{0}^{h}\right), q^{h}\right), \quad \forall q^{h} \in S^{h} .
\end{aligned}
$$


The update $\left(\boldsymbol{\sigma}_{1}^{h}, \mathbf{u}_{1}^{h}, p_{1}^{h}\right):=\left(\boldsymbol{\sigma}_{0}^{h}+\boldsymbol{\xi}_{0}^{h}, \mathbf{u}_{0}^{h}+\boldsymbol{\epsilon}_{0}^{h}, p_{0}^{h}+\rho_{0}^{h}\right)$ is expected to be a better approximation to $\left(\boldsymbol{\sigma}^{h}, \mathbf{u}^{h}, p^{h}\right)$ than $\left(\boldsymbol{\sigma}_{0}^{h}, \mathbf{u}_{0}^{h}, p_{0}^{h}\right)$. Note that combining (4.1)-(4.3) with (4.4)-(4.6), ( $\left.\boldsymbol{\sigma}_{1}^{h}, \mathbf{u}_{1}^{h}, p_{1}^{h}\right)$ satisfies:

$$
\begin{gathered}
\left.\left(\boldsymbol{\sigma}_{1}^{h}, \boldsymbol{\tau}^{h}\right)+\bar{\lambda} B^{h}\left(\mathbf{u}_{0}^{h}, \boldsymbol{\sigma}_{1}^{h}, \boldsymbol{\tau}^{h}\right)+\widetilde{\lambda}\left(g_{a}\left(\boldsymbol{\sigma}_{1}^{h}, \nabla \mathbf{u}_{0}^{h}\right), \boldsymbol{\tau}^{h}\right)-2 \alpha\left(\mathbf{D}\left(\mathbf{u}_{1}^{h}\right), \boldsymbol{\tau}^{h}\right)\right) \\
\quad=-(\lambda-\bar{\lambda}) B^{h}\left(\mathbf{u}_{0}^{h}, \boldsymbol{\sigma}_{0}^{h}, \boldsymbol{\tau}^{h}\right)-(\lambda-\widetilde{\lambda})\left(g_{a}\left(\boldsymbol{\sigma}_{0}^{h}, \nabla \mathbf{u}_{0}^{h}\right), \boldsymbol{\tau}^{h}\right), \quad \forall \boldsymbol{\tau}^{h} \in \boldsymbol{\Sigma}^{h}, \\
\left(\boldsymbol{\sigma}_{1}^{h}, \mathbf{D}\left(\mathbf{v}^{h}\right)\right)+2(1-\alpha)\left(\mathbf{D}\left(\mathbf{u}_{1}^{h}\right), \mathbf{D}\left(\mathbf{v}^{h}\right)\right)-\left(p_{1}^{h}, \nabla \cdot \mathbf{v}^{h}\right)=\left(\mathbf{f}, \mathbf{v}^{h}\right), \quad \forall \mathbf{v}^{h} \in \mathbf{X}^{h}, \\
\left(q^{h}, \nabla \cdot \mathbf{u}_{1}^{h}\right)=0, \quad \forall q^{h} \in S^{h} .
\end{gathered}
$$

The second step can be repeated with $\left(\boldsymbol{\sigma}_{0}^{h}, \mathbf{u}_{0}^{h}, p_{0}^{h}\right)$ replaced by $\left(\boldsymbol{\sigma}_{1}^{h}, \mathbf{u}_{1}^{h}, p_{1}^{h}\right)$ to further improve the approximation. The defect-correction method is summarized in Algorithm 4.1.

\section{Algorithm 4.1 (Defect-Correction Method for the Johnson-Segalman model)}

Step 1: Solve the nonlinear defected problem: Find $\left(\boldsymbol{\sigma}_{0}^{h}, \mathbf{u}_{0}^{h}, p_{0}^{h}\right) \in \boldsymbol{\Sigma}^{h} \times \mathbf{X}^{h} \times S^{h}$ such that

$$
\begin{aligned}
& \left(\boldsymbol{\sigma}_{0}^{h}, \boldsymbol{\tau}^{h}\right)+\bar{\lambda} B^{h}\left(\mathbf{u}_{0}^{h}, \boldsymbol{\sigma}_{0}^{h}, \boldsymbol{\tau}^{h}\right)+\widetilde{\lambda}\left(g_{a}\left(\boldsymbol{\sigma}_{0}^{h}, \nabla \mathbf{u}_{0}^{h}\right), \boldsymbol{\tau}^{h}\right)-2 \alpha\left(\mathbf{D}\left(\mathbf{u}_{0}^{h}\right), \boldsymbol{\tau}^{h}\right)=0, \quad \forall \boldsymbol{\tau}^{h} \in \boldsymbol{\Sigma}^{h}, \\
& \left(\boldsymbol{\sigma}_{0}^{h}, \mathbf{D}\left(\mathbf{v}^{h}\right)\right)+2(1-\alpha)\left(\mathbf{D}\left(\mathbf{u}_{0}^{h}\right), \mathbf{D}\left(\mathbf{v}^{h}\right)\right)-\left(p_{0}^{h}, \nabla \cdot \mathbf{v}^{h}\right)=\left(\mathbf{f}, \mathbf{v}^{h}\right), \quad \forall \mathbf{v}^{h} \in \mathbf{X}^{h}, \\
& \left(q^{h}, \nabla \cdot \mathbf{u}_{0}^{h}\right)=0, \quad \forall q^{h} \in S^{h},
\end{aligned}
$$

where $\bar{\lambda}$ and $\tilde{\lambda}$ are chosen to be less than or equal to $\lambda$.

Step 2: For $i=0,1,2, \ldots$, solve the following problem for the correction: Find $\left(\boldsymbol{\sigma}_{i+1}^{h}, \mathbf{u}_{i+1}^{h}, p_{i+1}^{h}\right) \in$ $\boldsymbol{\Sigma}^{h} \times \mathbf{X}^{h} \times S^{h}$ such that

$$
\begin{aligned}
& \left.\left(\boldsymbol{\sigma}_{i+1}^{h}, \boldsymbol{\tau}^{h}\right)+\bar{\lambda} B^{h}\left(\mathbf{u}_{i}^{h}, \boldsymbol{\sigma}_{i+1}^{h}, \boldsymbol{\tau}^{h}\right)+\widetilde{\lambda}\left(g_{a}\left(\boldsymbol{\sigma}_{i+1}^{h}, \nabla \mathbf{u}_{i}^{h}\right), \boldsymbol{\tau}^{h}\right)-2 \alpha\left(\mathbf{D}\left(\mathbf{u}_{i+1}^{h}\right), \boldsymbol{\tau}^{h}\right)\right) \\
& =-(\lambda-\bar{\lambda}) B^{h}\left(\mathbf{u}_{i}^{h}, \boldsymbol{\sigma}_{i}^{h}, \boldsymbol{\tau}^{h}\right)-(\lambda-\widetilde{\lambda})\left(g_{a}\left(\boldsymbol{\sigma}_{i}^{h}, \nabla \mathbf{u}_{i}^{h}\right), \boldsymbol{\tau}^{h}\right), \quad \forall \boldsymbol{\tau}^{h} \in \boldsymbol{\Sigma}^{h}, \\
& \left(\boldsymbol{\sigma}_{i+1}^{h}, \mathbf{D}\left(\mathbf{v}^{h}\right)\right)+2(1-\alpha)\left(\mathbf{D}\left(\mathbf{u}_{i+1}^{h}\right), \mathbf{D}\left(\mathbf{v}^{h}\right)\right)-\left(p_{i+1}^{h}, \nabla \cdot \mathbf{v}^{h}\right)=\left(\mathbf{f}, \mathbf{v}^{h}\right), \quad \forall \mathbf{v}^{h} \in \mathbf{X}^{h}, \\
& \left(q^{h}, \nabla \cdot \mathbf{u}_{i+1}^{h}\right)=0, \quad \forall q^{h} \in S^{h} .
\end{aligned}
$$

\subsection{Analysis of the Defect Step}

Assume $(\boldsymbol{\sigma}, \mathbf{u}, p)$ is an exact solution of (2.5)-(2.7) (the undefected continuous problem). Also let $(\overline{\boldsymbol{\sigma}}, \overline{\mathbf{u}}, \bar{p})$ be an exact solution of (2.5)-(2.7) with the first $\lambda$ replaced with $\bar{\lambda}$ and the second $\lambda$ replaced with $\widetilde{\lambda}$ (the defected continuous problem). Let $M$ be given by

$$
M:=\max \left\{\|\boldsymbol{\sigma}\|_{2},\|\mathbf{u}\|_{3},\|p\|_{2},\|\overline{\boldsymbol{\sigma}}\|_{2}\|\overline{\mathbf{u}}\|_{3},\|\bar{p}\|_{2}\right\} .
$$

Lemma 4.1 Let $\bar{\lambda}=\lambda-K_{1} h^{3 / 2}$ and $\tilde{\lambda}=\lambda-K_{2} h^{3 / 2}$ where $0 \leq K_{1} h^{3 / 2}, K_{2} h^{3 / 2} \leq \lambda$. If there exists an $M$ such that the undefected continuous problem (2.5)-(2.7) admits a bounded solution $(\boldsymbol{\sigma}, \mathbf{u}, p) \in \mathbf{H}^{2}(\Omega) \times \mathbf{H}^{3}(\Omega) \times\left(H^{2}(\Omega) \cap L_{0}^{2}(\Omega)\right)$, then for $h$ sufficiently small, for all $h \leq h_{0}$, then Step 1 of Algorithm 4.1 admits a solution $\left(\boldsymbol{\sigma}_{0}^{h}, \mathbf{u}_{0}^{h}, p_{0}^{h}\right) \in \boldsymbol{\Sigma}^{h} \times \mathbf{X}^{h} \times S^{h}$ and there exists a constant $C_{1}$ such that

$$
\left\|\boldsymbol{\sigma}-\boldsymbol{\sigma}_{0}^{h}\right\|_{0}+\left\|\nabla\left(\mathbf{u}-\mathbf{u}_{0}^{h}\right)\right\|_{0} \leq C_{1} h^{3 / 2} .
$$


Proof: From the embedding properties of Sobolev spaces [27] there exists a constant $C_{M}$ such that

$$
\begin{aligned}
\|\mathbf{v}\|_{0,4} & \leq C_{M}\|\nabla \mathbf{v}\|_{0,2}, \quad \forall \mathbf{v} \in \mathbf{H}_{0}^{1}(\Omega), \\
\|\mathbf{v}\|_{0, \infty} & \leq C_{M}\|\mathbf{v}\|_{2,2}, \quad \forall \mathbf{v} \in \mathbf{H}^{2}(\Omega) .
\end{aligned}
$$

Existence of a solution to Step 1 is shown by Baranger and Sandri [19]. For the proof of (4.7), let $(\overline{\boldsymbol{\sigma}}, \overline{\mathbf{u}}, \bar{p})$ be a solution of (2.5)-(2.7) with the first $\lambda$ replaced with $\bar{\lambda}$ and the second $\lambda$ replaced with $\widetilde{\lambda}$. Then, from [19], we have that

$$
\left\|\overline{\boldsymbol{\sigma}}-\boldsymbol{\sigma}_{0}^{h}\right\|_{0}+\left\|\nabla\left(\overline{\mathbf{u}}-\mathbf{u}_{0}^{h}\right)\right\|_{0} \leq \tilde{C} h^{3 / 2},
$$

for some positive constant $\tilde{C}$. Note that the solution $(\overline{\boldsymbol{\sigma}}, \overline{\mathbf{u}})$ satisfies the equations

$$
\begin{aligned}
& (\overline{\boldsymbol{\sigma}}, \boldsymbol{\tau})+\bar{\lambda}(\overline{\mathbf{u}} \cdot \nabla) \overline{\boldsymbol{\sigma}}, \boldsymbol{\tau})+\widetilde{\lambda}\left(g_{a}(\overline{\boldsymbol{\sigma}}, \nabla \overline{\mathbf{u}}), \boldsymbol{\tau}\right)-2 \alpha(\mathbf{D}(\overline{\mathbf{u}}), \boldsymbol{\tau})=0, \quad \forall \boldsymbol{\tau} \in \boldsymbol{\Sigma}, \\
& (\overline{\boldsymbol{\sigma}}, \mathbf{D}(\mathbf{v}))+2(1-\alpha)(\mathbf{D}(\overline{\mathbf{u}}), \mathbf{D}(\mathbf{v}))=(\mathbf{f}, \mathbf{v}), \quad \forall \mathbf{v} \in \mathbf{V} .
\end{aligned}
$$

To show the stated result, we obtain an estimation for $\|\nabla(\mathbf{u}-\overline{\mathbf{u}})\|_{0}$ and $\|\boldsymbol{\sigma}-\overline{\boldsymbol{\sigma}}\|_{0}$. In fact, as shown in Lemma 4.2 of [28], we have

$$
\|\boldsymbol{\sigma}-\overline{\boldsymbol{\sigma}}\|_{0}^{2}+\|\nabla(\mathbf{u}-\overline{\mathbf{u}})\|_{0}^{2} \leq C_{0}^{2} h^{3}
$$

where

$$
C_{0}^{2}=M^{4} C_{M}^{2}\left(K_{1} C_{M}+8 K_{2}\right)^{2} / \min \left\{\frac{3}{4}-\frac{M}{2}\left(\bar{\lambda} C_{M}^{2}+24 \widetilde{\lambda} C_{M}\right), 4 \alpha(1-\alpha)-\frac{M}{2}\left(\bar{\lambda} C_{M}^{2}+8 \widetilde{\lambda} C_{M}\right)\right\} .
$$

Thus, (4.7) follows from (4.9) and (4.10).

\subsection{Analysis of the Correction Step}

Step 1 of Algorithm 4.1 produces an initial approximation $\left(\boldsymbol{\sigma}_{0}^{h}, \mathbf{u}_{0}^{h}\right) \in \boldsymbol{\Sigma}^{h} \times \mathbf{V}^{h}$ that is the solution of the stable defected problem. This approximation is within a certain radius of the solution to the undefected problem. Step 2 of the algorithm provides an iterative procedure for correcting this initial iterate to an approximate solution of the original undefected problem. When convergent the computed approximation $\left(\boldsymbol{\sigma}^{h}, \mathbf{u}^{h}\right)$ will satisfy the same error estimates shown by Baranger and Sandri [19], namely (4.7) with $\left(\boldsymbol{\sigma}_{0}^{h}, \mathbf{u}_{0}^{h}\right)$ replaced by $\left(\boldsymbol{\sigma}^{h}, \mathbf{u}^{h}\right)$.

Let the nonempty ball $\mathcal{B}_{h} \subset \boldsymbol{\Sigma}^{h} \times \mathbf{V}^{h}$, centered at $(\boldsymbol{\sigma}, \mathbf{u})$, be defined by

$$
\mathcal{B}_{h}=\left\{\left(\boldsymbol{\tau}^{h}, \mathbf{v}^{h}\right) \in \boldsymbol{\Sigma}^{h} \times \mathbf{V}^{h}:\left\|\boldsymbol{\sigma}-\boldsymbol{\tau}^{h}\right\|_{0},\left\|\nabla\left(\mathbf{u}-\mathbf{v}^{h}\right)\right\|_{0} \leq \bar{C} h^{3 / 2}\right\}
$$

To show Step 2 of the algorithm converges, we construct a mapping $\Phi: \mathcal{B}_{h} \rightarrow \boldsymbol{\Sigma}^{h} \times \mathbf{V}^{h}$ such that $\Phi\left(\boldsymbol{\sigma}_{i}^{h}, \mathbf{u}_{i}^{h}\right)=\left(\boldsymbol{\sigma}_{i+1}^{h}, \mathbf{u}_{i+1}^{h}\right)$ where $\left(\boldsymbol{\sigma}_{i+1}^{h}, \mathbf{u}_{i+1}^{h}\right)$ satisfies

$$
\begin{gathered}
\left(\boldsymbol{\sigma}_{i+1}^{h}, \boldsymbol{\tau}^{h}\right)+\bar{\lambda} B^{h}\left(\mathbf{u}_{i}^{h}, \boldsymbol{\sigma}_{i+1}^{h}, \boldsymbol{\tau}^{h}\right)+\widetilde{\lambda}\left(g_{a}\left(\boldsymbol{\sigma}_{i+1}^{h}, \nabla \mathbf{u}_{i}^{h}\right), \boldsymbol{\tau}^{h}\right)-2 \alpha\left(\mathbf{D}\left(\mathbf{u}_{i+1}^{h}\right), \boldsymbol{\tau}^{h}\right) \\
=-(\lambda-\bar{\lambda}) B^{h}\left(\mathbf{u}_{i}^{h}, \boldsymbol{\sigma}_{i}^{h}, \boldsymbol{\tau}^{h}\right)-(\lambda-\widetilde{\lambda})\left(g_{a}\left(\boldsymbol{\sigma}_{i}^{h}, \nabla \mathbf{u}_{i}^{h}\right), \boldsymbol{\tau}^{h}\right) \quad \forall \boldsymbol{\tau}^{h} \in \boldsymbol{\Sigma}^{h}, \\
\left(\boldsymbol{\sigma}_{i+1}^{h}, \mathbf{D}\left(\mathbf{v}^{h}\right)\right)+2(1-\alpha)\left(\mathbf{D}\left(\mathbf{u}_{i+1}^{h}\right), \mathbf{D}\left(\mathbf{v}^{h}\right)\right)=\left(\mathbf{f}, \mathbf{v}^{h}\right) \quad \forall \mathbf{v}^{h} \in \mathbf{V}^{h} .
\end{gathered}
$$


The proof is similar in structure to that in [19] for a different iteration operator $\Phi$ and consists of three parts,

1. Show $\Phi$ is well defined and bounded on bounded sets.

2. Show $\Phi$ is continuous.

3. Show that $\Phi\left(\mathcal{B}_{h}\right) \subset \mathcal{B}_{h}$.

Then Brouwer's fixed-point theorem guarantees the existence of a fixed point $\left(\boldsymbol{\sigma}^{h}, \mathbf{u}^{h}\right) \in \mathcal{B}_{h}$ of $\Phi$ satisfying

$$
\left\|\boldsymbol{\sigma}-\boldsymbol{\sigma}^{h}\right\|_{0}+\left\|\nabla\left(\mathbf{u}-\mathbf{u}^{h}\right)\right\|_{0} \leq C h^{3 / 2}
$$

We begin with some preliminary bounds.

Lemma 4.2 Let $\mathbf{u}_{0}^{h}$ satisfy

$$
\left\|\nabla\left(\mathbf{u}-\mathbf{u}_{0}^{h}\right)\right\|_{0} \leq C_{0} h^{3 / 2}
$$

for some constant $C_{0}$. Then the quantities $\left\|\mathbf{u}_{0}^{h}\right\|_{\infty}$ and $\left\|\nabla \mathbf{u}_{0}^{h}\right\|_{\infty}$ are bounded.

Proof: Note that if $\tilde{\mathbf{u}}^{h} \in \mathbf{V}^{h}$ is defined by

$$
\left(\nabla\left(\mathbf{u}-\tilde{\mathbf{u}}^{h}\right), \nabla \mathbf{v}^{h}\right)=0 \quad \forall \mathbf{v}^{h} \in \mathbf{V}^{h},
$$

then standard approximation results ([20]) imply that

$$
\left\|\nabla\left(\mathbf{u}-\tilde{\mathbf{u}}^{h}\right)\right\|_{0} \leq C h^{2}\|\mathbf{u}\|_{3}
$$

and

$$
\left\|\nabla\left(\mathbf{u}-\tilde{\mathbf{u}}^{h}\right)\right\|_{1,4} \leq C h\|\mathbf{u}\|_{2,4},
$$

for some constant $C$. Using (3.14), (4.14), (4.15) and the imbedding theorem of $W^{1,4}$ in $L^{\infty}, H^{1}$ in $L^{4}$, we have

$$
\begin{aligned}
\left\|\mathbf{u}_{0}^{h}\right\|_{\infty} & \leq\|\mathbf{u}\|_{\infty}+\left\|\mathbf{u}-\tilde{\mathbf{u}}^{h}\right\|_{\infty}+\left\|\tilde{\mathbf{u}}^{h}-\mathbf{u}_{0}^{h}\right\|_{\infty} \\
& \leq C\left[M+\left\|\mathbf{u}-\tilde{\mathbf{u}}^{h}\right\|_{1,4}+h^{-1 / 2}\left\|\tilde{\mathbf{u}}^{h}-\mathbf{u}_{0}^{h}\right\|_{0,4}\right] \\
& \leq C\left[M+h\|\mathbf{u}\|_{2,4}+h^{-1 / 2}\left(\left\|\nabla\left(\tilde{\mathbf{u}}^{h}-\mathbf{u}\right)\right\|_{0}+\left\|\nabla\left(\mathbf{u}-\mathbf{u}_{0}^{h}\right)\right\|_{0}\right)\right] \\
& \leq C\left[M+M h+M h^{3 / 2}+C_{0} h\right] .
\end{aligned}
$$

The boundedness of $\left\|\nabla \mathbf{u}_{0}^{h}\right\|_{\infty}$ is established similarly.

Note that we can write (4.12)-(4.13) as

$$
\tilde{A}\left(\mathbf{u}_{i}^{h},\left(\boldsymbol{\sigma}_{i+1}^{h}, \mathbf{u}_{i+1}^{h}\right),\left(\boldsymbol{\tau}^{h}, \mathbf{v}^{h}\right)\right)+\bar{\lambda} B^{h}\left(\mathbf{u}_{i}^{h}, \boldsymbol{\sigma}_{i+1}^{h}, \boldsymbol{\tau}^{h}\right)=F\left(\left(\boldsymbol{\sigma}_{i}^{h}, \mathbf{u}_{i}^{h}\right),\left(\boldsymbol{\tau}^{h}, \mathbf{v}^{h}\right)\right)
$$


where

$$
\begin{aligned}
\tilde{A}\left(\mathbf{u}_{i}^{h},\left(\boldsymbol{\sigma}_{i+1}^{h}, \mathbf{u}_{i+1}^{h}\right),\left(\boldsymbol{\tau}^{h}, \mathbf{v}^{h}\right)\right)= & \left(\boldsymbol{\sigma}_{i+1}^{h}, \boldsymbol{\tau}^{h}\right)+\widetilde{\lambda}\left(g_{a}\left(\boldsymbol{\sigma}_{i+1}^{h}, \nabla \mathbf{u}_{i}^{h}\right), \boldsymbol{\tau}^{h}\right)-2 \alpha\left(\mathbf{D}\left(\mathbf{u}_{i+1}^{h}\right), \boldsymbol{\tau}^{h}\right) \\
& +2 \alpha\left(\boldsymbol{\sigma}_{i+1}^{h}, \mathbf{D}\left(\mathbf{v}^{h}\right)\right)+4 \alpha(1-\alpha)\left(\mathbf{D}\left(\mathbf{u}_{i+1}^{h}\right), \mathbf{D}\left(\mathbf{v}^{h}\right)\right), \\
B^{h}\left(\mathbf{u}_{i}^{h}, \boldsymbol{\sigma}_{i+1}^{h}, \boldsymbol{\tau}^{h}\right)= & \left(\left(\mathbf{u}_{i}^{h} \cdot \nabla\right) \boldsymbol{\sigma}_{i+1}^{h}, \boldsymbol{\tau}^{h}\right)_{h}+\frac{1}{2}\left(\nabla \cdot \mathbf{u}_{i}^{h} \boldsymbol{\sigma}_{i+1}^{h}, \boldsymbol{\tau}^{h}\right) \\
& +\left\langle\boldsymbol{\sigma}_{i+1}^{h^{+}}-\boldsymbol{\sigma}_{i+1}^{h^{-}}, \boldsymbol{\tau}^{h+}\right\rangle_{h, \mathbf{u}_{i}^{h}}, \\
F\left(\left(\boldsymbol{\sigma}_{i}^{h}, \mathbf{u}_{i}^{h}\right),\left(\boldsymbol{\tau}^{h}, \mathbf{v}^{h}\right)\right)= & 2 \alpha\left(\mathbf{f}, \mathbf{v}^{h}\right)-(\lambda-\bar{\lambda}) B^{h}\left(\mathbf{u}_{i}^{h}, \boldsymbol{\sigma}_{i}^{h}, \boldsymbol{\tau}^{h}\right) \\
& -(\lambda-\tilde{\lambda})\left(g_{a}\left(\boldsymbol{\sigma}_{i}^{h}, \nabla \mathbf{u}_{i}^{h}\right), \boldsymbol{\tau}^{h}\right) .
\end{aligned}
$$

Now we will discuss the three properties for $\Phi$. For the proof of the three properties for all $i=0,1, \ldots$, it is sufficient to show each property holds for $i=0$. The reader is referred to [28] for most of the technical details of the proofs to follow.

Lemma 4.3 Assume $M$ and $\widetilde{\lambda}$ satisfy

$$
1-4 \widetilde{\lambda} C^{*} M>0
$$

where $C^{*}$ is given by

$$
\left\|\nabla \mathbf{u}_{0}^{h}\right\|_{\infty} \leq C\left(2 M+M h+\bar{C} h^{1 / 2}\right) \leq C^{*} M .
$$

Then $\Phi$ is well-defined and bounded on bounded sets.

Proof: Proof of the coercivity of $\tilde{A}$ and $B^{h}$, and that $\Phi$ is bounded on bounded sets is shown in Lemma 4.4 of [28]. In particular, we have

$$
\tilde{A}\left(\mathbf{u}_{0}^{h},\left(\boldsymbol{\sigma}_{1}^{h}, \mathbf{u}_{1}^{h}\right),\left(\boldsymbol{\sigma}_{1}^{h}, \mathbf{u}_{1}^{h}\right)\right) \geq\left(1-4 \widetilde{\lambda} C^{*} M\right)\left\|\boldsymbol{\sigma}_{1}^{h}\right\|_{0}^{2}+4 \alpha(1-\alpha)\left\|\mathbf{D}\left(\mathbf{u}_{1}^{h}\right)\right\|_{0}^{2} .
$$

Lemma 4.4 Assume $1-4 \bar{\lambda} C^{*} M>0$. Then $\Phi$ is continuous on $\mathcal{B}_{h}$.

Proof: To show $\Phi$ is continuous, we want to show that if $\Phi\left(\boldsymbol{\sigma}_{0}^{h}, \mathbf{u}_{0}^{h}\right)=\left(\boldsymbol{\sigma}_{1}^{h}, \mathbf{u}_{1}^{h}\right)$ and $\Phi\left(\boldsymbol{\tau}_{0}^{h}, \mathbf{v}_{0}^{h}\right)=$ $\left(\boldsymbol{\tau}_{1}^{h}, \mathbf{v}_{1}^{h}\right)$, then

$$
\left\|\left(\boldsymbol{\sigma}_{1}^{h}-\boldsymbol{\tau}_{1}^{h}, \mathbf{u}_{1}^{h}-\mathbf{v}_{1}^{h}\right)\right\|_{\boldsymbol{\Sigma}^{h} \times \mathbf{V}^{h}} \leq C \eta\left(h, \boldsymbol{\sigma}_{0}^{h}, \boldsymbol{\tau}_{0}^{h}, \mathbf{u}_{0}^{h}, \mathbf{v}_{0}^{h}\right),
$$

where

$$
\lim _{\left(\boldsymbol{\tau}_{0}^{h}, \mathbf{v}_{0}^{h}\right) \rightarrow\left(\boldsymbol{\sigma}_{0}^{h}, \mathbf{u}_{0}^{h}\right)} \eta\left(h, \boldsymbol{\sigma}_{0}^{h}, \boldsymbol{\tau}_{0}^{h}, \mathbf{u}_{0}^{h}, \mathbf{v}_{0}^{h}\right)=0 .
$$

For ease of notation the superscript $h$ is suppressed on the variables $\boldsymbol{\sigma}^{h}, \boldsymbol{\tau}^{h}, \mathbf{u}^{h}, \mathbf{v}^{h}$ in the remainder of this proof. Now $\Phi\left(\boldsymbol{\sigma}_{0}, \mathbf{u}_{0}\right)=\left(\boldsymbol{\sigma}_{1}, \mathbf{u}_{1}\right)$ implies

$$
\tilde{A}\left(\mathbf{u}_{0},\left(\boldsymbol{\sigma}_{1}, \mathbf{u}_{1}\right),(\boldsymbol{\tau}, \mathbf{v})\right)+\bar{\lambda} B^{h}\left(\mathbf{u}_{0}, \boldsymbol{\sigma}_{1}, \boldsymbol{\tau}\right)=F\left(\left(\boldsymbol{\sigma}_{0}, \mathbf{u}_{0}\right),(\boldsymbol{\tau}, \mathbf{v})\right), \quad \forall(\boldsymbol{\tau}, \mathbf{v}) \in \mathcal{B}_{h}
$$


and $\Phi\left(\boldsymbol{\tau}_{0}, \mathbf{v}_{0}\right)=\left(\boldsymbol{\tau}_{1}, \mathbf{v}_{1}\right)$ implies

$$
\tilde{A}\left(\mathbf{v}_{0},\left(\boldsymbol{\tau}_{1}, \mathbf{v}_{1}\right),(\boldsymbol{\tau}, \mathbf{v})\right)+\bar{\lambda} B^{h}\left(\mathbf{v}_{0}, \boldsymbol{\tau}_{1}, \boldsymbol{\tau}\right)=F\left(\left(\boldsymbol{\tau}_{0}, \mathbf{v}_{0}\right),(\boldsymbol{\tau}, \mathbf{v})\right), \quad \forall(\boldsymbol{\tau}, \mathbf{v}) \in \mathcal{B}_{h} .
$$

Subtracting (4.19) from (4.18) and rearranging we obtain

$$
\begin{aligned}
\tilde{A}\left(\mathbf{u}_{0},\left(\boldsymbol{\sigma}_{1}-\boldsymbol{\tau}_{1}, \mathbf{u}_{1}-\mathbf{v}_{1}\right),(\boldsymbol{\tau}, \mathbf{v})\right)+ & \bar{\lambda} B^{h}\left(\mathbf{u}_{0}, \boldsymbol{\sigma}_{1}-\boldsymbol{\tau}_{1}, \boldsymbol{\tau}\right) \\
= & -(\lambda-\bar{\lambda})\left[B^{h}\left(\mathbf{u}_{0}, \boldsymbol{\sigma}_{0}, \boldsymbol{\tau}\right)-B^{h}\left(\mathbf{v}_{0}, \boldsymbol{\tau}_{0}, \boldsymbol{\tau}\right)\right] \\
& -(\lambda-\widetilde{\lambda})\left[\left(g_{a}\left(\boldsymbol{\sigma}_{0}, \nabla \mathbf{u}_{0}\right), \boldsymbol{\tau}\right)-\left(g_{a}\left(\boldsymbol{\tau}_{0}, \nabla \mathbf{v}_{0}\right), \boldsymbol{\tau}\right)\right] \\
& -\bar{\lambda}\left[B^{h}\left(\mathbf{u}_{0}, \boldsymbol{\tau}_{1}, \boldsymbol{\tau}\right)-B^{h}\left(\mathbf{v}_{0}, \boldsymbol{\tau}_{1}, \boldsymbol{\tau}\right)\right] \\
& -\widetilde{\lambda}\left(g_{a}\left(\boldsymbol{\tau}_{1}, \nabla\left(\mathbf{u}_{0}-\mathbf{v}_{0}\right)\right), \boldsymbol{\tau}\right), \quad \forall(\boldsymbol{\tau}, \mathbf{v}) \in \mathcal{B}_{h} .
\end{aligned}
$$

Let $\boldsymbol{\tau}=\boldsymbol{\sigma}_{1}-\boldsymbol{\tau}_{1}$ and $\mathbf{v}=\mathbf{u}_{1}-\mathbf{v}_{1}$. Then (4.20), (4.17) and the assumption on $1-4 \widetilde{\lambda} C^{*} M$ implies

$$
\begin{aligned}
C\left\|\left(\boldsymbol{\sigma}_{1}-\boldsymbol{\tau}_{1}, \mathbf{u}_{1}-\mathbf{v}_{1}\right)\right\|_{\boldsymbol{\Sigma}^{h} \times \mathbf{V}^{h}}^{2} \leq & -(\lambda-\bar{\lambda})\left[B^{h}\left(\mathbf{u}_{0}, \boldsymbol{\sigma}_{0}, \boldsymbol{\sigma}_{1}-\boldsymbol{\tau}_{1}\right)-B^{h}\left(\mathbf{v}_{0}, \boldsymbol{\tau}_{0}, \boldsymbol{\sigma}_{1}-\boldsymbol{\tau}_{1}\right)\right] \\
& -(\lambda-\widetilde{\lambda})\left[\left(g_{a}\left(\boldsymbol{\sigma}_{0}, \nabla \mathbf{u}_{0}\right), \boldsymbol{\sigma}_{1}-\boldsymbol{\tau}_{1}\right)-\left(g_{a}\left(\boldsymbol{\tau}_{0}, \nabla \mathbf{v}_{0}\right), \boldsymbol{\sigma}_{1}-\boldsymbol{\tau}_{1}\right)\right] \\
& -\bar{\lambda}\left[B^{h}\left(\mathbf{u}_{0}, \boldsymbol{\tau}_{1}, \boldsymbol{\sigma}_{1}-\boldsymbol{\tau}_{1}\right)-B^{h}\left(\mathbf{v}_{0}, \boldsymbol{\tau}_{1}, \boldsymbol{\sigma}_{1}-\boldsymbol{\tau}_{1}\right)\right] \\
& -\widetilde{\lambda}\left(g_{a}\left(\boldsymbol{\tau}_{1}, \nabla\left(\mathbf{u}_{0}-\mathbf{v}_{0}\right)\right), \boldsymbol{\sigma}_{1}-\boldsymbol{\tau}_{1}\right) .
\end{aligned}
$$

For notational simplicity, let

$$
\hat{\boldsymbol{\sigma}}_{0}=\boldsymbol{\sigma}_{0}-\boldsymbol{\tau}_{0}, \quad \hat{\boldsymbol{\sigma}}_{1}=\boldsymbol{\sigma}_{1}-\boldsymbol{\tau}_{1}, \quad \hat{\mathbf{u}}_{0}=\mathbf{u}_{0}-\mathbf{v}_{0}, \quad \hat{\mathbf{u}}_{1}=\mathbf{u}_{1}-\mathbf{v}_{1} .
$$

Then (4.21) can be written as

$$
\begin{aligned}
C\left\|\left(\hat{\boldsymbol{\sigma}}_{1}, \hat{\mathbf{u}}_{1}\right)\right\|_{\boldsymbol{\Sigma}^{h} \times \mathbf{V}^{h}}^{2} \leq & -(\lambda-\bar{\lambda})\left[B^{h}\left(\mathbf{u}_{0}, \hat{\boldsymbol{\sigma}}_{0}, \hat{\boldsymbol{\sigma}}_{1}\right)+B^{h}\left(\mathbf{u}_{0}, \boldsymbol{\tau}_{0}, \hat{\boldsymbol{\sigma}}_{1}\right)-B^{h}\left(\mathbf{v}_{0}, \boldsymbol{\tau}_{0}, \hat{\boldsymbol{\sigma}}_{1}\right)\right] \\
& -(\lambda-\tilde{\lambda})\left[\left(g_{a}\left(\boldsymbol{\sigma}_{0}, \nabla \mathbf{u}_{0}\right), \hat{\boldsymbol{\sigma}}_{1}\right)-\left(g_{a}\left(\boldsymbol{\tau}_{0}, \nabla \mathbf{v}_{0}\right), \hat{\boldsymbol{\sigma}}_{1}\right)\right] \\
& -\bar{\lambda}\left[B^{h}\left(\mathbf{u}_{0}, \boldsymbol{\tau}_{1}, \hat{\boldsymbol{\sigma}}_{1}\right)-B^{h}\left(\mathbf{v}_{0}, \boldsymbol{\tau}_{1}, \hat{\boldsymbol{\sigma}}_{1}\right)\right]-\widetilde{\lambda}\left(g_{a}\left(\boldsymbol{\tau}_{1}, \nabla \hat{\mathbf{u}}_{0}\right), \hat{\boldsymbol{\sigma}}_{1}\right) .
\end{aligned}
$$

Each of the terms on the right-hand side of (4.22) can be shown to go to zero as $\left(\boldsymbol{\tau}_{0}, \mathbf{v}_{0}\right)$ goes to $\left(\boldsymbol{\sigma}_{0}, \mathbf{u}_{0}\right)$, for a fixed $h$ (Lemma 4.5 of [28]). Thus $\Phi$ is continuous on $\mathcal{B}_{h}$.

The proof that $\Phi\left(\mathcal{B}_{h}\right) \subset \mathcal{B}_{h}$ is similar to the proof of Theorem 4.1 in [7]. Several labelled constants are used in the data conditions below, the reader is referred to Lemma 4.6 of [28] for their origin.

Lemma 4.5 There exists constants $C$ and $\delta$ such that if $h$ and $M \leq \bar{C}$ are small enough to satisfy (4.16),

$$
\begin{aligned}
& \bar{C} h^{1 / 2} \leq M \\
& C^{\prime} M \leq \bar{C} / 4
\end{aligned}
$$

and

$$
\frac{C}{\delta}\left(\frac{2(2 \bar{\lambda} M+\widetilde{\lambda} M)^{2}}{\left(1-4 \widetilde{\lambda} C^{*} M\right)}+\frac{h}{2 \alpha(1-\alpha)}+\bar{\lambda} M\right)^{1 / 2} \leq 1
$$

then $\Phi\left(\mathcal{B}_{h}\right) \subset \mathcal{B}_{h}$. 
Proof: If $\tilde{\boldsymbol{\sigma}}^{h} \in \boldsymbol{\Sigma}^{h}$ is the orthogonal projection of $\boldsymbol{\sigma}$ on $T^{h}$ in $\boldsymbol{\Sigma}$, and $\tilde{\mathbf{u}}^{h} \in \mathbf{V}^{h}$ is defined by

$$
\left(\nabla\left(\mathbf{u}-\tilde{\mathbf{u}}^{h}\right), \nabla \mathbf{v}^{h}\right)=0 \quad \forall \mathbf{v}^{h} \in \mathbf{V}^{h},
$$

then standard approximation results $([20])$ imply that there exists a constant $C^{\prime}$ such that

$$
\begin{aligned}
\left\|\nabla\left(\mathbf{u}-\tilde{\mathbf{u}}^{h}\right)\right\|_{0} & \leq C^{\prime} h^{2}\|\mathbf{u}\|_{3}, \\
\left\|\boldsymbol{\sigma}-\tilde{\boldsymbol{\sigma}}^{h}\right\|_{0}+h\left\|\nabla\left(\boldsymbol{\sigma}-\tilde{\boldsymbol{\sigma}}^{h}\right)\right\|_{0} & \leq C^{\prime} h^{2}\|\boldsymbol{\sigma}\|_{2}, \\
\left\|\boldsymbol{\sigma}-\tilde{\boldsymbol{\sigma}}^{h}\right\|_{0, \Gamma^{h}} & \leq C^{\prime} h^{3 / 2}\|\boldsymbol{\sigma}\|_{2} .
\end{aligned}
$$

Assuming $h<1$ and $C^{\prime} M \leq \bar{C} / 4$ guarantees that

$$
\left\|\boldsymbol{\sigma}-\tilde{\boldsymbol{\sigma}}^{h}\right\|_{0}+\left\|\nabla\left(\mathbf{u}-\tilde{\mathbf{u}}^{h}\right)\right\|_{0} \leq \frac{\bar{C}}{2} h^{2} \leq \frac{\bar{C}}{2} h^{3 / 2},
$$

thus $\left(\tilde{\boldsymbol{\sigma}}^{h}, \tilde{\mathbf{u}}^{h}\right) \in \mathcal{B}_{h}$.

To prove $\Phi\left(\mathcal{B}_{h}\right) \subset \mathcal{B}_{h}$, we show that, given $\left(\boldsymbol{\sigma}_{0}^{h}, \mathbf{u}_{0}^{h}\right) \in \mathcal{B}_{h}, \Phi\left(\boldsymbol{\sigma}_{0}^{h}, \mathbf{u}_{0}^{h}\right)=\left(\boldsymbol{\sigma}_{1}^{h}, \mathbf{u}_{1}^{h}\right) \in \mathcal{B}_{h}$. Let $\left(\boldsymbol{\sigma}_{0}^{h}, \mathbf{u}_{0}^{h}\right) \in \mathcal{B}_{h}$. Then,

$$
\left\|\boldsymbol{\sigma}-\boldsymbol{\sigma}_{0}^{h}\right\|_{0}+\left\|\nabla\left(\mathbf{u}-\mathbf{u}_{0}^{h}\right)\right\|_{0} \leq \bar{C} h^{3 / 2}
$$

and Lemma 4.2 implies that

$$
\left\|\mathbf{u}_{0}^{h}\right\|_{\infty} \leq C\left[M+M h+M h^{3 / 2}+\bar{C} h\right]
$$

and

$$
\left\|\nabla \mathbf{u}_{0}^{h}\right\|_{\infty} \leq C\left[2 M+M h+\bar{C} h^{1 / 2}\right] .
$$

The assumption of $\bar{C} h^{1 / 2} \leq M$ then implies the existence of a constant $C_{u}$ such that

$$
\left\|\mathbf{u}_{0}^{h}\right\|_{\infty} \leq C_{u} M \quad \text { and } \quad\left\|\nabla \mathbf{u}_{0}^{h}\right\|_{\infty} \leq C_{u} M .
$$

Since $(\boldsymbol{\sigma}, \mathbf{u}, p)$ satisfies $(2.5)-(2.7)$, we have

$$
\begin{aligned}
A\left((\boldsymbol{\sigma}, \mathbf{u}),\left(\boldsymbol{\tau}^{h}, \mathbf{v}^{h}\right)\right)+\lambda\left(\mathbf{u} \cdot \nabla \boldsymbol{\sigma}, \boldsymbol{\tau}^{h}\right)+\lambda\left(g_{a}(\boldsymbol{\sigma}, \nabla \mathbf{u}), \boldsymbol{\tau}^{h}\right)-\left(p, \nabla \cdot \mathbf{v}^{h}\right) & \\
& =2 \alpha\left(\mathbf{f}, \mathbf{v}^{h}\right), \quad \forall\left(\boldsymbol{\tau}^{h}, \mathbf{v}^{h}\right) \in \boldsymbol{\Sigma}^{h} \times \mathbf{V}^{h} .
\end{aligned}
$$

and, since $\nabla \cdot \mathbf{u}=0$ and $\boldsymbol{\sigma}$ is continuous

$$
B^{h}\left(\mathbf{u}, \boldsymbol{\sigma}, \boldsymbol{\tau}^{h}\right)=\left((\mathbf{u} \cdot \nabla) \boldsymbol{\sigma}, \boldsymbol{\tau}^{h}\right)_{h}=\left((\mathbf{u} \cdot \nabla) \boldsymbol{\sigma}, \boldsymbol{\tau}^{h}\right) .
$$

Also, $B^{h}(\cdot, \boldsymbol{\sigma}, \cdot)$ can be treated as a bilinear form (since the jump term vanishes when the second argument in $B^{h}(\cdot, \cdot, \cdot)$ is continuous $)$. Now $\left(\boldsymbol{\sigma}_{1}^{h}, \mathbf{u}_{1}^{h}\right)=\Phi\left(\boldsymbol{\sigma}_{0}^{h}, \mathbf{u}_{0}^{h}\right)$ satisfies the relation

$$
\begin{aligned}
A\left(\left(\boldsymbol{\sigma}_{1}^{h}, \mathbf{u}_{1}^{h}\right),\left(\boldsymbol{\tau}^{h}, \mathbf{v}^{h}\right)\right)+\bar{\lambda} B^{h}\left(\mathbf{u}_{0}^{h}, \boldsymbol{\sigma}_{1}^{h}, \boldsymbol{\tau}^{h}\right)+\widetilde{\lambda}\left(g_{a}\left(\boldsymbol{\sigma}_{1}^{h}, \nabla \mathbf{u}_{0}^{h}\right), \boldsymbol{\tau}^{h}\right) \\
=2 \alpha(\mathbf{f}, \mathbf{v})-(\lambda-\bar{\lambda}) B^{h}\left(\mathbf{u}_{0}^{h}, \boldsymbol{\sigma}_{0}^{h}, \boldsymbol{\tau}^{h}\right) \\
\quad-(\lambda-\tilde{\lambda})\left(g_{a}\left(\boldsymbol{\sigma}_{0}^{h}, \nabla \mathbf{u}_{0}^{h}\right), \boldsymbol{\tau}^{h}\right), \quad \forall\left(\boldsymbol{\tau}^{h}, \mathbf{v}^{h}\right) \in \boldsymbol{\Sigma}^{h} \times \mathbf{V}^{h} .
\end{aligned}
$$


Subtracting (4.27) from (4.26) yields

$$
\begin{aligned}
& A\left(\left(\boldsymbol{\sigma}-\boldsymbol{\sigma}_{1}^{h}, \mathbf{u}-\mathbf{u}_{1}^{h}\right),\left(\boldsymbol{\tau}^{h}, \mathbf{v}^{h}\right)\right) \\
& +\bar{\lambda}\left(B^{h}\left(\mathbf{u}, \boldsymbol{\sigma}, \boldsymbol{\tau}^{h}\right)-B^{h}\left(\mathbf{u}_{0}^{h}, \boldsymbol{\sigma}_{1}^{h}, \boldsymbol{\tau}^{h}\right)\right)+(\lambda-\bar{\lambda}) B^{h}\left(\mathbf{u}, \boldsymbol{\sigma}, \boldsymbol{\tau}^{h}\right) \\
& +\widetilde{\lambda}\left(\left(g_{a}(\boldsymbol{\sigma}, \nabla \mathbf{u}), \boldsymbol{\tau}^{h}\right)-\left(g_{a}\left(\boldsymbol{\sigma}_{1}^{h}, \nabla \mathbf{u}_{0}^{h}\right), \boldsymbol{\tau}^{h}\right)\right) \\
& \quad+(\lambda-\widetilde{\lambda})\left(g_{a}(\boldsymbol{\sigma}, \nabla \mathbf{u}), \boldsymbol{\tau}^{h}\right)-\left(p, \nabla \cdot \mathbf{v}^{h}\right) \\
& =(\lambda-\bar{\lambda}) B^{h}\left(\mathbf{u}_{0}^{h}, \boldsymbol{\sigma}_{0}^{h}, \boldsymbol{\tau}^{h}\right)+(\lambda-\widetilde{\lambda})\left(g_{a}\left(\boldsymbol{\sigma}_{0}^{h}, \nabla \mathbf{u}_{0}^{h}\right), \boldsymbol{\tau}^{h}\right), \quad \forall\left(\boldsymbol{\tau}^{h}, \mathbf{v}^{h}\right) \in \boldsymbol{\Sigma}^{h} \times \mathbf{V}^{h} .
\end{aligned}
$$

i.e.,

$$
\begin{aligned}
A\left(\left(\boldsymbol{\sigma}-\tilde{\boldsymbol{\sigma}}^{h}, \mathbf{u}-\tilde{\mathbf{u}}^{h}\right),\left(\boldsymbol{\tau}^{h}, \mathbf{v}^{h}\right)\right)+A\left(\left(\tilde{\boldsymbol{\sigma}}^{h}-\boldsymbol{\sigma}_{1}^{h}, \tilde{\mathbf{u}}^{h}-\mathbf{u}_{1}^{h}\right),\left(\boldsymbol{\tau}^{h}, \mathbf{v}^{h}\right)\right) \\
+\bar{\lambda}\left(B^{h}\left(\mathbf{u}, \boldsymbol{\sigma}, \boldsymbol{\tau}^{h}\right)+B^{h}\left(\mathbf{u}_{0}^{h}, \tilde{\boldsymbol{\sigma}}^{h}-\boldsymbol{\sigma}_{1}^{h}, \boldsymbol{\tau}^{h}\right)-B^{h}\left(\mathbf{u}_{0}^{h}, \tilde{\boldsymbol{\sigma}}^{h}, \boldsymbol{\tau}^{h}\right)\right)+(\lambda-\bar{\lambda}) B^{h}\left(\mathbf{u}, \boldsymbol{\sigma}, \boldsymbol{\tau}^{h}\right) \\
\left.\quad+\widetilde{\lambda}\left(\left(g_{a}(\boldsymbol{\sigma}, \nabla \mathbf{u}), \boldsymbol{\tau}^{h}\right)+g_{a}\left(\tilde{\boldsymbol{\sigma}}^{h}-\boldsymbol{\sigma}_{1}^{h}, \nabla \mathbf{u}_{0}^{h}\right), \boldsymbol{\tau}^{h}\right)-\left(g_{a}\left(\tilde{\boldsymbol{\sigma}}^{h}, \nabla \mathbf{u}_{0}^{h}\right), \boldsymbol{\tau}^{h}\right)\right) \\
\quad+(\lambda-\tilde{\lambda})\left(g_{a}(\boldsymbol{\sigma}, \nabla \mathbf{u}), \boldsymbol{\tau}^{h}\right)-\left(p, \nabla \cdot \mathbf{v}^{h}\right) \\
\quad(\lambda-\bar{\lambda}) B^{h}\left(\mathbf{u}_{0}^{h}, \boldsymbol{\sigma}_{0}^{h}, \boldsymbol{\tau}^{h}\right)+(\lambda-\widetilde{\lambda})\left(g_{a}\left(\boldsymbol{\sigma}_{0}^{h}, \nabla \mathbf{u}_{0}^{h}\right), \boldsymbol{\tau}^{h}\right), \quad \forall\left(\boldsymbol{\tau}^{h}, \mathbf{v}^{h}\right) \in \boldsymbol{\Sigma}^{h} \times \mathbf{V}^{h} .
\end{aligned}
$$

Rearranging (4.28) we obtain

$$
\begin{aligned}
A\left(\left(\tilde{\boldsymbol{\sigma}}^{h}-\boldsymbol{\sigma}_{1}^{h}, \tilde{\mathbf{u}}^{h}\right.\right. & \left.\left.-\mathbf{u}_{1}^{h}\right),\left(\boldsymbol{\tau}^{h}, \mathbf{v}^{h}\right)\right) \\
& +\bar{\lambda} B^{h}\left(\mathbf{u}_{0}^{h}, \tilde{\boldsymbol{\sigma}}^{h}-\boldsymbol{\sigma}_{1}^{h}, \boldsymbol{\tau}^{h}\right)+\widetilde{\lambda}\left(g_{a}\left(\tilde{\boldsymbol{\sigma}}^{h}-\boldsymbol{\sigma}_{1}^{h}, \nabla \mathbf{u}_{0}^{h}\right), \boldsymbol{\tau}^{h}\right) \\
= & -A\left(\left(\boldsymbol{\sigma}-\tilde{\boldsymbol{\sigma}}^{h}, \mathbf{u}-\tilde{\mathbf{u}}^{h}\right),\left(\boldsymbol{\tau}^{h}, \mathbf{v}^{h}\right)\right) \\
& +\bar{\lambda}\left(B^{h}\left(\mathbf{u}_{0}^{h}, \tilde{\boldsymbol{\sigma}}^{h}, \boldsymbol{\tau}^{h}\right)-B^{h}\left(\mathbf{u}, \boldsymbol{\sigma}, \boldsymbol{\tau}^{h}\right)\right) \\
& +\tilde{\lambda}\left(\left(g_{a}\left(\tilde{\boldsymbol{\sigma}}^{h}, \nabla \mathbf{u}_{0}^{h}\right), \boldsymbol{\tau}^{h}\right)-\left(g_{a}(\boldsymbol{\sigma}, \nabla \mathbf{u}), \boldsymbol{\tau}^{h}\right)\right) \\
& +(\lambda-\bar{\lambda})\left(B^{h}\left(\mathbf{u}_{0}^{h}, \boldsymbol{\sigma}_{0}^{h}, \boldsymbol{\tau}^{h}\right)-B^{h}\left(\mathbf{u}, \boldsymbol{\sigma}, \boldsymbol{\tau}^{h}\right)\right) \\
& +(\lambda-\tilde{\lambda})\left(\left(g_{a}\left(\boldsymbol{\sigma}_{0}^{h}, \nabla \mathbf{u}_{0}^{h}\right), \boldsymbol{\tau}^{h}\right)-\left(g_{a}(\boldsymbol{\sigma}, \nabla \mathbf{u}), \boldsymbol{\tau}^{h}\right)\right) \\
& +\left(p-q^{h}, \nabla \cdot \mathbf{v}^{h}\right), \quad \forall\left(\boldsymbol{\tau}^{h}, \mathbf{v}^{h}, q^{h}\right) \in \boldsymbol{\Sigma}^{h} \times \mathbf{V}^{h} \times S^{h}
\end{aligned}
$$

Let $\boldsymbol{\tau}^{h}=\tilde{\boldsymbol{\sigma}}^{h}-\boldsymbol{\sigma}_{1}^{h}$ and $\mathbf{v}^{h}=\tilde{\mathbf{u}}^{h}-\mathbf{u}_{1}^{h}$. For ease of notation, let $\hat{\boldsymbol{\sigma}}=\tilde{\boldsymbol{\sigma}}^{h}-\boldsymbol{\sigma}_{1}^{h}$ and $\hat{\mathbf{u}}=\tilde{\mathbf{u}}^{h}-\mathbf{u}_{1}^{h}$. 
Using (3.7) and Lemma 4.3, from (4.8) and (4.29) we have

$$
\begin{aligned}
&\left(1-4 \widetilde{\lambda} C^{*} M\right)\|\hat{\boldsymbol{\sigma}}\|_{0}^{2}+4 \alpha(1-\alpha)\|\nabla \hat{\mathbf{u}}\|_{0}^{2}+\frac{\bar{\lambda}}{2}\left\langle\left\langle\hat{\boldsymbol{\sigma}}^{+}-\hat{\boldsymbol{\sigma}}^{-}\right\rangle\right\rangle_{h, \mathbf{u}_{0}^{h}}^{2} \\
& \leq \tilde{A}\left(\mathbf{u}_{0}^{h},(\hat{\boldsymbol{\sigma}}, \hat{\mathbf{u}}),(\hat{\boldsymbol{\sigma}}, \hat{\mathbf{u}})\right)+\bar{\lambda} B^{h}\left(\mathbf{u}_{0}^{h}, \hat{\boldsymbol{\sigma}}, \hat{\boldsymbol{\sigma}}\right) \\
&=-A\left(\left(\boldsymbol{\sigma}-\tilde{\boldsymbol{\sigma}}^{h}, \mathbf{u}-\tilde{\mathbf{u}}^{h}\right),(\hat{\boldsymbol{\sigma}}, \hat{\mathbf{u}})\right) \\
&+\bar{\lambda}\left(B^{h}\left(\mathbf{u}_{0}^{h}, \tilde{\boldsymbol{\sigma}}^{h}, \hat{\boldsymbol{\sigma}}\right)-B^{h}(\mathbf{u}, \boldsymbol{\sigma}, \hat{\boldsymbol{\sigma}})\right) \\
&+\tilde{\lambda}\left(\left(g_{a}\left(\tilde{\boldsymbol{\sigma}}^{h}, \nabla \mathbf{u}_{0}^{h}\right), \hat{\boldsymbol{\sigma}}\right)-\left(g_{a}(\boldsymbol{\sigma}, \nabla \mathbf{u}), \hat{\boldsymbol{\sigma}}\right)\right) \\
&+(\lambda-\bar{\lambda})\left(B^{h}\left(\mathbf{u}_{0}^{h}, \boldsymbol{\sigma}_{0}^{h}, \hat{\boldsymbol{\sigma}}\right)-B^{h}(\mathbf{u}, \boldsymbol{\sigma}, \hat{\boldsymbol{\sigma}})\right) \\
&+(\lambda-\tilde{\lambda})\left(\left(g_{a}\left(\boldsymbol{\sigma}_{0}^{h}, \nabla \mathbf{u}_{0}^{h}\right), \hat{\boldsymbol{\sigma}}\right)-\left(g_{a}(\boldsymbol{\sigma}, \nabla \mathbf{u}), \hat{\boldsymbol{\sigma}}\right)\right) \\
&+\left(p-q^{h}, \nabla \cdot \hat{\mathbf{u}}\right) .
\end{aligned}
$$

Each of the terms in the right-hand side above can be bounded (see [28] for details). From (4.98) of [28], we have

$$
\begin{aligned}
\left(1-4 \widetilde{\lambda} C^{*} M\right)\|\hat{\boldsymbol{\sigma}}\|_{0}^{2}+ & 4 \alpha(1-\alpha)\|\nabla \hat{\mathbf{u}}\|_{0}^{2}+\frac{\bar{\lambda}}{2}\left\langle\left\langle\hat{\boldsymbol{\sigma}}^{+}-\hat{\boldsymbol{\sigma}}^{-}\right\rangle\right\rangle_{h, \mathbf{u}_{0}^{h}}^{2} \\
\leq & \tilde{A}\left(\mathbf{u}_{0}^{h},(\hat{\boldsymbol{\sigma}}, \hat{\mathbf{u}}),(\hat{\boldsymbol{\sigma}}, \hat{\mathbf{u}})\right)+\bar{\lambda} B^{h}\left(\mathbf{u}_{0}^{h}, \hat{\boldsymbol{\sigma}}, \hat{\boldsymbol{\sigma}}\right) \\
\leq & \left(C_{3} \bar{\lambda}\left(\bar{C} M+M^{2}\right)+C_{4} \widetilde{\lambda} \bar{C} M\right) h^{3 / 2}\|\hat{\boldsymbol{\sigma}}\|_{0} \\
& +\left(C_{2}+C_{7}\right) M h^{2}\|\nabla \hat{\mathbf{u}}\|_{0} \\
& +C_{3} \bar{\lambda} C_{u}^{1 / 2} M^{3 / 2} h^{3 / 2}\left\langle\left\langle\hat{\boldsymbol{\sigma}}^{+}-\hat{\boldsymbol{\sigma}}^{-}\right\rangle\right\rangle_{h, \mathbf{u}_{0}^{h}}
\end{aligned}
$$

Thus (4.30), (4.16), Young's inequality, and $M \leq \bar{C}$ implies

$$
\begin{aligned}
& \frac{1}{2}\left(1-4 \widetilde{\lambda} C^{*} M\right)\|\hat{\boldsymbol{\sigma}}\|_{0}^{2}+2 \alpha(1-\alpha)\|\nabla \hat{\mathbf{u}}\|_{0}^{2}+\frac{\bar{\lambda}}{4}\left\langle\left\langle\hat{\boldsymbol{\sigma}}^{+}-\hat{\boldsymbol{\sigma}}^{-}\right\rangle\right\rangle_{h, \mathbf{u}_{0}^{h}}^{2} \\
& \leq\left(\frac{2\left(2 C_{3} \bar{\lambda} M+C_{4} \widetilde{\lambda} M\right)^{2}}{\left(1-4 \widetilde{\lambda} C^{*} M\right)}+\frac{h\left(C_{2}+C_{7}\right)^{2}}{2 \alpha(1-\alpha)}+\bar{\lambda}\left(C_{3} C_{u}^{1 / 2} M^{1 / 2}\right)^{2}\right) \frac{\bar{C}^{2}}{4} h^{3} .
\end{aligned}
$$

Let $\delta$ be given by

$$
2 \delta^{2}=\min \left\{\frac{1}{2}\left(1-4 \tilde{\lambda} C^{*} M\right), 2 \alpha(1-\alpha)\right\} .
$$

Then, (4.31) implies

$$
\begin{aligned}
\left(\sqrt{2} \delta\|\hat{\boldsymbol{\sigma}}\|_{0}\right)^{2} & +\left(\sqrt{2} \delta\|\nabla \hat{\mathbf{u}}\|_{0}\right)^{2} \\
& \leq\left(\frac{2\left(2 C_{3} \bar{\lambda} M+C_{4} \widetilde{\lambda} M\right)^{2}}{\left(1-4 \widetilde{\lambda} C^{*} M\right)}+\frac{h\left(C_{2}+C_{7}\right)^{2}}{2 \alpha(1-\alpha)}+\bar{\lambda}\left(C_{3} C_{u}^{1 / 2} M^{1 / 2}\right)^{2}\right) \frac{\bar{C}^{2}}{4} h^{3},
\end{aligned}
$$


i.e.,

$$
\delta\|\hat{\boldsymbol{\sigma}}\|_{0}+\delta\|\nabla \hat{\mathbf{u}}\|_{0} \leq\left(\frac{2\left(2 C_{3} \bar{\lambda} M+C_{4} \widetilde{\lambda} M\right)^{2}}{\left(1-4 \widetilde{\lambda} C^{*} M\right)}+\frac{h\left(C_{2}+C_{7}\right)^{2}}{2 \alpha(1-\alpha)}+\bar{\lambda}\left(C_{3} C_{u}^{1 / 2} M^{1 / 2}\right)^{2}\right)^{1 / 2} \frac{\bar{C}}{2} h^{3 / 2} .
$$

Hence, for $M$ and $h$ sufficiently small such that

$$
\frac{C}{\delta}\left(\frac{2(2 \bar{\lambda} M+\widetilde{\lambda} M)^{2}}{\left(1-4 \widetilde{\lambda} C^{*} M\right)}+\frac{h}{2 \alpha(1-\alpha)}+\bar{\lambda} M\right)^{1 / 2} \leq 1
$$

we have

$$
\|\hat{\boldsymbol{\sigma}}\|_{0}+\|\nabla \hat{\mathbf{u}}\|_{0} \leq \frac{\bar{C}}{2} h^{3 / 2}
$$

Thus (4.25) implies

$\left\|\boldsymbol{\sigma}-\boldsymbol{\sigma}_{1}^{h}\right\|_{0}+\left\|\nabla\left(\mathbf{u}-\mathbf{u}_{1}^{h}\right)\right\|_{0} \leq\|\hat{\boldsymbol{\sigma}}\|_{0}+\|\nabla \hat{\mathbf{u}}\|_{0}+\left\|\boldsymbol{\sigma}-\tilde{\boldsymbol{\sigma}}^{h}\right\|_{0}+\left\|\nabla\left(\mathbf{u}-\tilde{\mathbf{u}}^{h}\right)\right\|_{0} \leq \frac{\bar{C}}{2} h^{3 / 2}+\frac{\bar{C}}{2} h^{3 / 2}=\bar{C} h^{3 / 2}$.

Hence $\Phi\left(\mathcal{B}_{h}\right) \subset \mathcal{B}_{h}$.

Thus we have the following result.

Theorem 4.1 Assume that $\left(\boldsymbol{\sigma}_{0}^{h}, \mathbf{u}_{0}^{h}\right)$ is the solution obtained after Step 1 of Algorithm 4.1. Then for $M$ and $h$ sufficiently small, there exists a fixed point $\left(\boldsymbol{\sigma}^{h}, \mathbf{u}^{h}\right)$ of Step 2 of Algorithm 4.1 that satisfies (3.11)-(3.12) and each of the iterates $\left(\boldsymbol{\sigma}_{i}^{h}, \mathbf{u}_{i}^{h}\right), i=1,2, \ldots$, satisfy

$$
\left\|\boldsymbol{\sigma}-\boldsymbol{\sigma}_{i}^{h}\right\|_{0}+\left\|\nabla\left(\mathbf{u}-\mathbf{u}_{i}^{h}\right)\right\|_{0} \leq C h^{3 / 2}
$$

for constant $C>0$.

Proof: If $M$ satisfies the small data condition of Lemma 4.1, the solution $\left(\boldsymbol{\sigma}_{0}^{h}, \mathbf{u}_{0}^{h}\right)$ of the defect step satisfies

$$
\left\|\boldsymbol{\sigma}-\boldsymbol{\sigma}_{0}^{h}\right\|_{0}+\left\|\nabla\left(\mathbf{u}-\mathbf{u}_{0}^{h}\right)\right\|_{0} \leq C_{0} h^{3 / 2}
$$

Then $\left(\boldsymbol{\sigma}_{0}^{h}, \mathbf{u}_{0}^{h}\right) \in \mathcal{B}_{h}$ where

$$
\mathcal{B}_{h}=\left\{\left(\boldsymbol{\tau}^{h}, \mathbf{v}^{h}\right) \in \boldsymbol{\Sigma}^{h} \times \mathbf{V}^{h}:\left\|\boldsymbol{\sigma}-\boldsymbol{\tau}^{h}\right\|_{0},\left\|\nabla\left(\mathbf{u}-\mathbf{v}^{h}\right)\right\|_{0} \leq C_{0} h^{3 / 2}\right\} .
$$

Then by Lemmas 4.3-4.5, the mapping $\Phi$ defined by (4.12)-(4.13) satisfies $\Phi\left(\mathcal{B}_{h}\right) \subset \mathcal{B}_{h}$, and Brouwer's Fixed-Point Theorem guarantees the existence of a fixed point $\left(\boldsymbol{\sigma}^{h}, \mathbf{u}^{h}\right) \in \mathcal{B}_{h}$ of $\Phi$ solving (3.11)-(3.12). 


\subsection{Newton Corrector Iteration}

The correction step (Step 2) in Algorithm 4.1 uses a linearized iteration in order to solve the original nonlinear problem. This linearization will be referred to as the Picard corrector, as some terms that are present in the "full" linearization are not present (precisely those which contain updated velocity and lagged stress). This full linearization is obtained from computing the Fréchet derivative of the original problem at a known value $\left(\boldsymbol{\sigma}_{i}^{h}, \mathbf{u}_{i}^{h}, p_{i}^{h}\right)$, which we called the Newton linearization. Within the context of the defect correction method, the Newton corrector iteration has of the form:

$$
\begin{gathered}
A\left(\left(\boldsymbol{\sigma}_{i+1}^{h}, \mathbf{u}_{i+1}^{h}\right),\left(\boldsymbol{\tau}^{h}, \mathbf{v}^{h}\right)\right)+\bar{\lambda} B^{h}\left(\mathbf{u}_{i}^{h}, \boldsymbol{\sigma}_{i+1}^{h}, \boldsymbol{\tau}^{h}\right)+\bar{\lambda} B^{h}\left(\mathbf{u}_{i+1}^{h}, \boldsymbol{\sigma}_{i}^{h}, \boldsymbol{\tau}^{h}\right) \\
+\widetilde{\lambda}\left(g_{a}\left(\boldsymbol{\sigma}_{i+1}^{h}, \nabla \mathbf{u}_{i}^{h}\right), \boldsymbol{\tau}^{h}\right)+\widetilde{\lambda}\left(g_{a}\left(\boldsymbol{\sigma}_{i}^{h}, \nabla \mathbf{u}_{i+1}^{h}\right), \boldsymbol{\tau}^{h}\right) \\
=2 \alpha\left(\mathbf{f}, \mathbf{v}^{h}\right)-(\lambda-2 \bar{\lambda}) B^{h}\left(\mathbf{u}_{i}^{h}, \boldsymbol{\sigma}_{i}^{h}, \boldsymbol{\tau}^{h}\right) \\
\quad-(\lambda-2 \widetilde{\lambda})\left(g_{a}\left(\boldsymbol{\sigma}_{i}^{h}, \nabla \mathbf{u}_{i}^{h}\right), \boldsymbol{\tau}^{h}\right) \quad \forall\left(\boldsymbol{\tau}^{h}, \mathbf{v}^{h}\right) \in \boldsymbol{\Sigma}^{h} \times \mathbf{V}^{h} .
\end{gathered}
$$

The Newton corrector is expected to converge faster than the Picard corrector. However, convergence of the Newton corrector has not been proven. In addition, the computational implementation of (4.32) is nontrivial; the term $B^{h}\left(\mathbf{u}_{i+1}^{h}, \boldsymbol{\sigma}_{i}^{h}, \boldsymbol{\tau}^{h}\right)$ requires the calculation of the jump of the lagged stress $\left(\boldsymbol{\sigma}_{i}^{h}\right)$ in the direction of an unknown velocity $\left(\mathbf{u}_{i+1}^{h}\right)$. This difficulty is avoided by modifying (4.32) to use $\mathbf{u}_{i}^{h}$ for the jump term in all occurrences of $B^{h}$.

\section{$5 \quad$ Numerical experiments}

To investigate the accuracy and effectiveness of the defect-correction method, computational experiments are designed to gauge these attributes. The method has been implemented using the finite element software package FreeFem++ [29] in 2-d. Linear systems are solved using the UMFPACK solver. As described in the theoretical analysis, continuous piecewise quadratic elements are used for velocity, continuous piecewise linears are used for pressure, and discontinuous piecewise linears are used for stress. The solution approach used for undefected nonlinear problems, as well as the nonlinear defect step of the defect-correction algorithm, is a standard Newton iteration scheme. Results obtained on two different problems are presented:

1. A nonphysical problem on a square domain that has a known analytic solution. Computations show that the method satisfies the theoretical spatial convergence rate and converges to the same solution as the standard undefected solution approach.

2. A commonly cited benchmark physical problem of a four-to-one planar contraction flow. Computations show that solutions computed under mesh refinement converge to a solution computed using the standard undefected solution approach on a very fine spatial mesh. Computations also show that the method is successful in computing solutions beyond the critical Weissenberg value.

The three different methods for computing solutions are labeled as:

- STD: A standard Newton nonlinear iteration for the undefected problem. 
- DCN: A defect-correction method with a standard Newton nonlinear iteration in the defect step and a standard Newton-like linearization in the correction step.

- DCP: A defect-correction method with a standard Newton nonlinear iteration in the defect step and a Picard-like linearization in the correction step.

\subsection{Example 1}

As in [8] and [30], $\Omega=[0,1] \times[0,1] \subset \mathbb{R}^{2}$, and chosen functions are added to the right-hand sides of (2.1)-(2.3) so that the true solution to the problem is given by

$$
\mathbf{u}=\left(\begin{array}{c}
\sin (\pi x) y(y-1) \\
\sin (x)(x-1) y \cos (\pi y / 2))
\end{array}\right), \quad p=\cos (2 \pi x) y(y-1), \quad \boldsymbol{\sigma}=2 \alpha \mathbf{D}(\mathbf{u}) .
$$

The parameters $\alpha, a$ in the equations are chosen as 0.5 and 0 , respectively. A stopping criterion of

$$
\left\|\mathbf{u}_{i}^{h}-\mathbf{u}_{i-1}^{h}\right\|_{\infty},\left\|\boldsymbol{\sigma}_{i}^{h}-\boldsymbol{\sigma}_{i-1}^{h}\right\|_{\infty} \leq 10^{-8}
$$

is used for the iterative nonlinear solver in both the undefected nonlinear solution approach and the defect step of the defect-correction algorithm. The linear correction step in the defect-correction algorithm is iterated to the same tolerance. The solution computed for $\lambda=4.0$ was used as the initial iterate in all cases.

Table 5.1 presents results for $\lambda=5.0$. Specifically, the errors results and mesh convergence rates obtained by the STD method and the DCP method for several values of $\bar{\lambda}$ and $\widetilde{\lambda}$ are given. The resulting errors for both methods are seen to be the same. It should be noted that the convergence rates obtained for the cases below are greater than the theoretical spatial convergence rate of $3 / 2$. As is expected, for larger defects $\lambda-\bar{\lambda}$ and $\lambda-\widetilde{\lambda}$, the nonlinear and linear corrector iteration counts increase.

Also of interest is how DCP performs in comparison to DCN. In all experiments where both correction steps converged, each method obtained the same error result. Very few cases were observed in which one corrector method converged while the other did not. When the methods did not converge, the Newton corrector was more likely to diverge while the Picard corrector would stagnate. In most cases, the Picard iteration required more correction steps for convergence than the Newton iteration, as anticipated. Table 5.2 presents iteration counts obtained by both methods on the mesh

$h=1 / 8$ for several combinations of $\bar{\lambda}$ and $\widetilde{\lambda}$. In this table, NNI represents the number of nonlinear iterations required in the defect step, NCS represents the number of linear correction steps, and an asterisk denotes that the particular method did not converge for the given parameters.

\subsection{Example 2}

Numerical simulations of viscoelastic flow through a planar or axisymmetric contraction have been widely studied (see [31] or Chapter 8 of [32]). Here the case of planar flow through a contraction geometry with a ratio of $4: 1$ with respect to upstream and downstream channel widths is considered. The contraction angle is a fixed $3 \pi / 2$ and the channel lengths are sufficiently long to impose a fully developed Poiseuille flow in the inflow and outflow channels. The geometry of the computational domain is illustrated in Figure 5.1. The lower left corner of the domain corresponds to $x=y=0$. 


\begin{tabular}{|c|c|c|c|c|c|c|}
\hline & $h$ & $\begin{array}{c}\text { STD } \\
\text { method }\end{array}$ & $\begin{array}{c}\text { DCP } \\
\bar{\lambda}=4.9 \\
\tilde{\lambda}=4.9\end{array}$ & $\begin{array}{c}\text { DCP } \\
\bar{\lambda}=4.5 \\
\widetilde{\lambda}=4.5\end{array}$ & $\begin{array}{c}\text { DCP } \\
\bar{\lambda}=4.0 \\
\tilde{\lambda}=4.0\end{array}$ & $\begin{array}{c}\text { Rate of } \\
\text { convergence }\end{array}$ \\
\hline \multirow{4}{*}{$\begin{array}{c}H^{1} \text { error } \\
\text { of } \mathbf{u}\end{array}$} & $\frac{1}{4}$ & $4.5902 \mathrm{E}-02$ & $4.5902 \mathrm{E}-02$ & $4.5902 \mathrm{E}-02$ & $4.5902 \mathrm{E}-02$ & \\
\hline & $\frac{1}{8}$ & $1.1289 \mathrm{E}-02$ & $1.1289 \mathrm{E}-02$ & $1.1289 \mathrm{E}-02$ & $1.1289 \mathrm{E}-02$ & 2.02 \\
\hline & $\frac{1}{16}$ & $2.8788 \mathrm{E}-03$ & $2.8788 \mathrm{E}-03$ & $2.8788 \mathrm{E}-03$ & $2.8788 \mathrm{E}-03$ & 1.97 \\
\hline & $\frac{1}{32}$ & $7.1002 \mathrm{E}-04$ & 7.1003E-04 & $7.1003 \mathrm{E}-04$ & $7.1003 \mathrm{E}-04$ & 2.02 \\
\hline \multirow{4}{*}{$\begin{array}{c}L^{2} \text { error } \\
\text { of } \boldsymbol{\sigma}\end{array}$} & $\frac{1}{4}$ & $5.5428 \mathrm{E}-02$ & $5.5429 \mathrm{E}-02$ & $5.5429 \mathrm{E}-02$ & $5.5429 \mathrm{E}-02$ & \\
\hline & $\frac{1}{8}$ & $1.4605 \mathrm{E}-02$ & $1.4605 \mathrm{E}-02$ & $1.4605 \mathrm{E}-02$ & $1.4605 \mathrm{E}-02$ & 1.92 \\
\hline & $\frac{1}{16}$ & 3.3900E-03 & 3.3903E-03 & $3.3903 \mathrm{E}-03$ & $3.3903 \mathrm{E}-03$ & 2.11 \\
\hline & $\frac{1}{32}$ & 7.6339E-04 & 7.6371E-04 & 7.6371E-04 & 7.6371E-04 & 2.15 \\
\hline \multirow{4}{*}{$\begin{array}{c}\text { Number of } \\
\text { nonlinear } \\
\text { iterations } \\
(\mathrm{NNI})\end{array}$} & $\frac{1}{4}$ & 8 & 8 & 7 & 7 & \\
\hline & $\frac{1}{8}$ & 7 & 7 & 7 & 8 & \\
\hline & $\frac{1}{16}$ & 6 & 6 & 7 & 8 & \\
\hline & $\frac{1}{32}$ & 6 & 6 & 7 & 9 & \\
\hline \multirow{4}{*}{$\begin{array}{c}\text { Number of } \\
\text { correction } \\
\text { steps } \\
(\mathrm{NCS})\end{array}$} & $\frac{1}{4}$ & & 21 & 22 & 25 & \\
\hline & $\frac{1}{8}$ & & 25 & 26 & 25 & \\
\hline & $\frac{1}{16}$ & & 38 & 39 & 40 & \\
\hline & $\frac{1}{32}$ & & 50 & 54 & 56 & \\
\hline
\end{tabular}

Table 5.1: Errors and convergence rates for $\lambda=5.0$ and selected values of $\bar{\lambda}, \widetilde{\lambda}$, defect correction method (Picard corrector).

\begin{tabular}{|cc||ccc|}
\hline \multicolumn{2}{|c|}{$\lambda=5.0$} & & NCS & NCS \\
$\bar{\lambda}$ & $\tilde{\lambda}$ & NNI & (Picard) & (Newton) \\
\hline 4.9 & 4.9 & 7 & 25 & 5 \\
4.5 & 4.5 & 7 & 26 & 9 \\
4 & 4 & 8 & 25 & 14 \\
3 & 3 & 8 & 129 & 44 \\
5 & 4.5 & 8 & 29 & 11 \\
4.5 & 5 & 8 & 27 & 12 \\
5 & 4 & 10 & 46 & 19 \\
4 & 5 & 9 & 28 & 29 \\
5 & 3 & 11 & 612 & 107 \\
3 & 5 & 12 & 81 & $*$ \\
4.5 & 4 & 8 & 35 & 16 \\
4 & 4.5 & 8 & 26 & 17 \\
\hline
\end{tabular}

Table 5.2: Iteration counts for Picard and Newton correctors, $h=1 / 8, \lambda=5.0$, selected values of $\bar{\lambda}$ and $\widetilde{\lambda}$

The factor $L$ is set to $1 / 4$ for these computations. On this domain the velocity boundary conditions are

$$
\mathbf{u}=\left[\begin{array}{c}
\frac{1}{32}\left(1-y^{2}\right) \\
0
\end{array}\right] \quad \text { on } \Gamma_{\text {in }}, \quad \mathbf{u}=\left[\begin{array}{c}
2\left(\frac{1}{16}-y^{2}\right) \\
0
\end{array}\right] \quad \text { on } \Gamma_{\text {out }} .
$$

Boundary conditions for $\boldsymbol{\sigma}$ must be specified on the inflow boundary. From the constitutive equation (2.1) and the velocity conditions (5.1), for $u_{1, y}=\partial u_{1} / \partial y$, we have

$$
\boldsymbol{\sigma}_{x x}=\frac{-\alpha \lambda(a+1) u_{1, y}^{2}}{\left(a^{2}-1\right) \lambda^{2} u_{1, y}^{2}-1}, \quad \boldsymbol{\sigma}_{x y}=\frac{-\alpha u_{1, y}}{\left(a^{2}-1\right) \lambda^{2} u_{1, y}^{2}-1}, \quad \boldsymbol{\sigma}_{y y}=\frac{-\alpha \lambda(a-1) u_{1, y}^{2}}{\left(a^{2}-1\right) \lambda^{2} u_{1, y}^{2}-1}, \quad \text { on } \Gamma_{\mathrm{in}} .
$$

Symmetry conditions are imposed on the bottom of the computational domain. The parameter $\alpha$ 


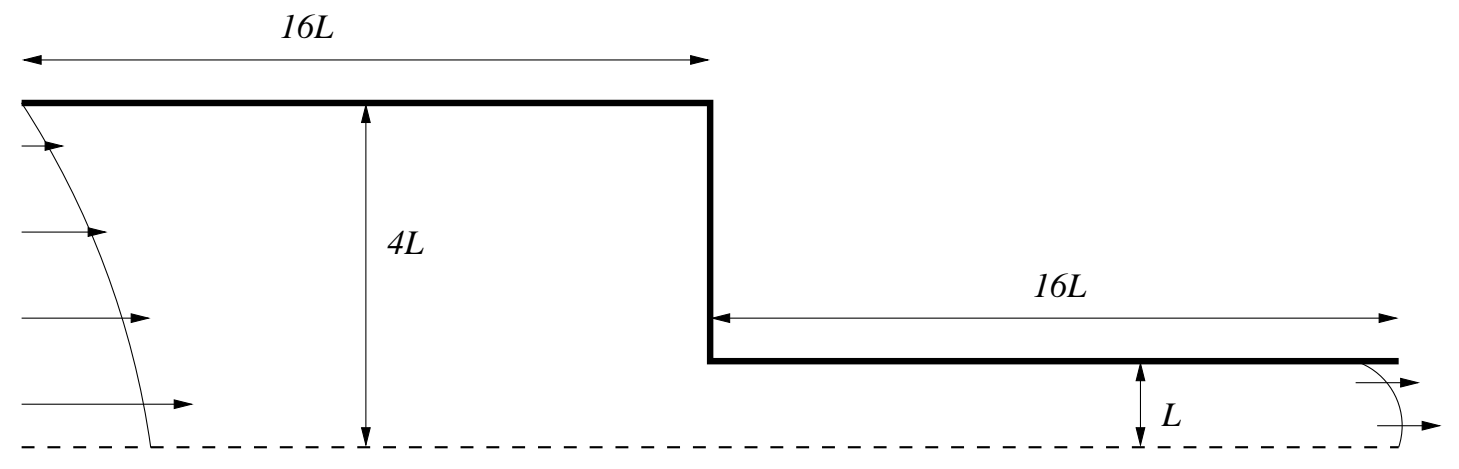

Figure 5.1: Geometry of 4:1 contraction domain

is set to $8 / 9$ and the initial iterate was given by $(\boldsymbol{\sigma}, \mathbf{u}, p)=(\mathbf{0}, \mathbf{0}, 0)$. Computations were performed on three different meshes, M1, M2, and M3. Table 5.3 lists the characteristics of the meshes.

\begin{tabular}{c|c|c|c} 
Mesh & $\Delta x_{\min }$ & $\Delta y_{\min }$ & No. of degrees of freedom \\
\hline M1 & 0.25 & 0.0625 & 1880 \\
M2 & 0.125 & 0.03125 & 7321 \\
M3 & 0.0625 & 0.015625 & 28895
\end{tabular}

Table 5.3: Mesh characteristics

To determine the accuracy of the defect-correction method, solutions computed on successive meshes were compared to a solution computed by the STD method on a very fine spatial mesh (114811 degrees of freedom). For the results shown here, $a=1$ (Oldroyd-B model) and $\lambda=0.7$. Table 5.4 gives values of solutions norms computed by the STD and DCP methods. As the mesh is refined, the norms of the computed approximations approach the values computed on the very fine mesh.

\begin{tabular}{|c|c|c|c|c|}
\hline Method & Mesh & $\left\|\mathbf{u}^{h}\right\|_{0}$ & $\left\|\mathbf{u}^{h}\right\|_{1}$ & $\left\|\boldsymbol{\sigma}^{h}\right\|_{0}$ \\
\hline STD & Fine & 0.104166 & 0.595209 & 0.932091 \\
\hline DCP & M1 & 0.104243 & 0.595417 & 0.935189 \\
$\bar{\lambda}=0.5$ & M2 & 0.104207 & 0.595287 & 0.933602 \\
$\widetilde{\lambda}=0.5$ & M3 & 0.104183 & 0.595243 & 0.932683 \\
\hline DCP & M1 & 0.104243 & 0.595417 & 0.935193 \\
$\bar{\lambda}=0.35$ & M2 & 0.104206 & 0.595287 & 0.933606 \\
$\widetilde{\lambda}=0.35$ & M3 & 0.104183 & 0.595243 & 0.932687 \\
\hline DCP & M1 & 0.104243 & 0.595417 & 0.935192 \\
$\bar{\lambda}=0.4$ & M2 & 0.104206 & 0.595287 & 0.933604 \\
$\widetilde{\lambda}=0.6$ & M3 & 0.104183 & 0.595243 & 0.932686 \\
\hline DCP & M1 & 0.104243 & 0.595417 & 0.935187 \\
$\bar{\lambda}=0.6$ & M2 & 0.104207 & 0.595287 & 0.933599 \\
$\widetilde{\lambda}=0.4$ & M3 & 0.104184 & 0.595243 & 0.932681 \\
\hline
\end{tabular}

Table 5.4: Solution norms, successive meshes, $\lambda=0.7, a=1$. 
For each method, $\lambda^{*}$ was the "maximum" value for which the method converged. The values of $\lambda^{*}$ obtained by STD, DCN, and DCP on both meshes for different values of $a$ are presented in Table 5.5. For the values of $a$ considered here, the $\lambda^{*}$-DCP values were the same as the $\lambda^{*}$-STD values for the case $a \neq 0$. However, DCN significantly improves the $\lambda^{*}$ values obtained by STD.

\begin{tabular}{c|c|c|c|c} 
Mesh & $a$ & $\lambda^{*}$-STD & $\lambda^{*}$-DCP & $\lambda^{*}$-DCN \\
\hline \multirow{3}{*}{ M1 } & 1.0 & 2.430 & 2.430 & 4.993 \\
& 0.0 & 1.286 & 2.722 & 2.719 \\
& -1.0 & 1.465 & 1.465 & 3.121 \\
\hline \multirow{3}{*}{ M2 } & 1.0 & 1.821 & 1.821 & 3.682 \\
& 0.0 & 0.934 & 1.990 & 1.993 \\
& -1.0 & 1.321 & 1.321 & 2.738 \\
\hline \multirow{3}{*}{ M3 } & 1.0 & 1.412 & 1.412 & 2.810 \\
& 0.0 & 0.823 & 1.666 & 1.663 \\
& -1.0 & 1.170 & 1.170 & 2.185
\end{tabular}

Table 5.5: Critical $\lambda$ values for $(\boldsymbol{\sigma}, \mathbf{u}, p)=(\mathbf{0}, \mathbf{0}, 0)$.

The two examples illustrate that the defect-correction approach can be effective in extending the range of Weissenberg numbers for which a numerical approximation to the viscoelastic fluid flow equations can be obtained. The optimal choices of the defect parameters is still an open question and is being investigated.

\section{References}

[1] K. Böhmer and H. J. Stetter. Defect Correction Methods - Theory and Applications. SpringerVerlag, Vienna, 1984.

[2] O. Axelsson and W. Layton. Defect correction methods for convection-dominated convectiondiffusion problems. RAIRO Modl. Math. Anal. Numr., 24:423-455, 1990.

[3] V. J. Ervin and W. J. Layton. A study of defect correction finite difference methods for convection dominated, convection diffusion equations. SIAM J. Numer. Anal., 26:169-179, 1989 .

[4] P. Hemker. Mixed defect correction iteration for the accurate solution of the convection diffusion equation. In W. Hackbusch and V. Trottenberg, editors, Multigrid Methods, Lecture Notes in Math., volume 960. Springer-Verlag, Berlin, 1982.

[5] W. Layton. Solution algorithm for incompressible viscous flows at high Reynolds number. Vestnik Mosk. Gos. Univ., Series, 15:25-35, 1996.

[6] H. Lee W. Layton and J. Peterson. A defect-correction method for the incompressible NavierStokes equations. Appl. Math. Comp., 129:1-19, 2002.

[7] H. Lee. Analysis of a defect correction method for viscoelastic fluid flow. Comput. Math. Appl., 48:1213-1229, 2004. 
[8] V. J. Ervin and H. Lee. Defect correction method for viscoelastic flows at high Weissenberg number. Numer. Meth. PDE, 22:145-164, 2006.

[9] H. B. Keller. Global homotopies and Newton methods. In C. deBoor and G. H. Golub, editors, Recent advances in numerical analysis, pages 73-94. Academic Press, New York, 1978.

[10] W. C. Rheinboldt. Solution fields of nonlinear equations and continuation methods. SIAM J. Numer. Anal., 17:221-237, 1980.

[11] E. L. Allgower and K. Georg. Numerical Continuation Methods: An Introduction, volume 13 of Springer Series in Computational Mathematics. Springer-Verlag, Berlin, 1990.

[12] C. Chauvière and R. G. Owens. A new spectral element method for the reliable computation of viscoelastic flow. Comput. Methods Appl. Mech. Engrg., 190:3999-4018, 2001.

[13] T. W. Chow. Continuation finite element analysis of viscoelastic fluids. PhD thesis, Colorado State University, Fort Collins, CO, 1987.

[14] A. R. Davies. Numerical filtering and the high Weissenberg number problem. J. Non-Newtonian Fluid Mech., 16:195-209, 1984.

[15] M. A. Mendelson, P.-W. Yeh, R. A. Brown, and R. C. Armstrong. Approximation error in finite element calculation of viscoelastic fluid flows. J. Non-Newtonian Fluid Mech., 10:31-54, 1982 .

[16] N. Pan-Thien and R. Zheng. On the continuous squeezing flow in a wedge. Rheol. Acta, 30:491-496, 1991.

[17] P.-W. Yeh, M. E. Kim-E, R. C. Armstrong, and R. A. Brown. Multiple solutions in the calculation of axisymmetric contraction flow of an upper convected Maxwell fluid. J. NonNewtonian Fluid Mech., 16:173-194, 1984.

[18] R. Zheng and N. Pan-Thien. On the non-orthogonal stagnation flow of the Oldroyd-B fluid. $Z$. A. M. P., 45:99-115, 1994.

[19] J. Baranger and D. Sandri. Finite element approximation of viscoelastic fluid flow: Existence of approximate solutions and error bounds. I. Discontinuous constraints. Numer. Math., 63:13-27, 1992.

[20] S. Brenner and L. Scott. The Mathematical Theory of Finite Element Methods. Springer-Verlag, 1994.

[21] M. Renardy. Mathematical Analysis of Viscoelastic Flows. CBMS-NSF Regional Conference Series in Applied Mathematics 73. Society for Industrial and Applied Mathematics, Philadelphia, 2000 .

[22] M. Renardy. Existence of slow steady flows of viscoelastic fluids with differential constitutive equations. Z.A.M.M., 65:449-451, 1985.

[23] C. Guillopé and J. C. Saut. Existence results for the flow of viscoelastic fluids with a differential constitutive law. Nonlinear Anal., 15(9), 1990. 
[24] E. Fernandez-Cara, F. Guillen, and R. R. Ortega. Mathematical modeling and analysis of viscoelastic fluids of the Oldroyd kind. In P. G. Ciarlet and J. L. Lions, editors, Handbooks of Numerical Analysis, Part 2, volume VIII, pages 543-661. North-Holland, Amsterdam, 2002.

[25] V. Girault and P. Raviart. Finite Element Methods for Navier-Stokes Equations. Springer, Berlin, 1986.

[26] C. Schwab. p- and hp-Finite Element Methods. Theory and applications to solid and fluid mechanics. Oxford University Press, 1998.

[27] R. A. Adams and J. J. F. Fournier. Sobolev Spaces, volume 140 of Pure and Applied Mathematics. Academic Press, 2003.

[28] V. J. Ervin, J. S. Howell, and H. Lee. Defect-correction strategies for viscoelastic fluid flow. Clemson University Mathematical Sciences Technical Report TR2006_10_EHL, 2006.

[29] F. Hecht, A. LeHyaric, and O. Pironneau. Freefem++ version 2.2-1, 2005.

[30] H. Lee. A multigrid method for viscoelastic fluid flow. SIAM J. Numer. Anal., 42:109-129, 2004 .

[31] F. P. T. Baaijens. Mixed finite element methods for viscoelastic flow analysis: A review. $J$. Non-Newtonian Fluid Mech., 79:361-385, 1998.

[32] R. G. Owens and T. N. Phillips. Computational Rheology. Imperial College Press, London, 2002 . 